\title{
Thematic role assignment in patients with Broca's aphasia: Sentence-picture matching electrified
}

\author{
Marlies Wassenaar $^{\mathrm{a}, \mathrm{b}, *}$, Peter Hagoort ${ }^{\mathrm{b}, *}$ \\ ${ }^{a}$ Max Planck Institute for Psycholinguistics, Nijmegen, The Netherlands \\ ${ }^{\mathrm{b}}$ F.C. Donders Centre for Cognitive Neuroimaging, Radboud University Nijmegen, Kapittelweg 29, \\ P.O. Box 9101, NL-6500 HB Nijmegen, The Netherlands
}

Received 7 March 2005; received in revised form 12 June 2006; accepted 6 August 2006

Available online 26 September 2006

\begin{abstract}
An event-related brain potential experiment was carried out to investigate on-line thematic role assignment during sentence-picture matching in patients with Broca's aphasia. Subjects were presented with a picture that was followed by an auditory sentence. The sentence either matched the picture or mismatched the visual information depicted. Sentences differed in complexity, and ranged from simple active semantically irreversible sentences to passive semantically reversible sentences. ERPs were recorded while subjects were engaged in sentence-picture matching. In addition, reaction time and accuracy were measured. Three groups of subjects were tested: Broca patients $(N=10)$, non-aphasic patients with a right hemisphere $(\mathrm{RH})$ lesion $(N=8)$, and healthy aged-matched controls $(N=15)$. The results of this study showed that, in neurologically unimpaired individuals, thematic role assignment in the context of visual information was an immediate process. This in contrast to patients with Broca's aphasia who demonstrated no signs of on-line sensitivity to the picture-sentence mismatches. The syntactic contribution to the thematic role assignment process seemed to be diminished given the reduction and even absence of P600 effects. Nevertheless, Broca patients showed some off-line behavioral sensitivity to the sentence-picture mismatches. The long response latencies of Broca's aphasics make it likely that off-line response strategies were used.
\end{abstract}

(C) 2006 Elsevier Ltd. All rights reserved.

Keywords: Broca's aphasia; Sentence-picture matching; Thematic role assignment; Event-related brain potentials; P600

\section{Introduction}

In patients with Broca's aphasia, the ability to understand what is spoken to them is relatively spared when compared to their sentence production. However, comprehension problems can typically emerge when the understanding of sentences hinges exclusively upon the correct analysis of syntactic structure (Caplan \& Hildebrandt, 1988). These comprehension difficulties especially arise when sentences are semantically reversible (Caramazza \& Zurif, 1976). A sentence is semantically reversible if, after major noun phrases have exchanged position, the sentence still makes sense:

(1) The lion is casing the tiger.

(2) The lion is eating the bone.

\footnotetext{
* Corresponding authors. Tel.: +31 2436 10750; fax: +31 243610652 .

E-mail addresses: marlies.wassenaar@fcdonders.ru.nl (M. Wassenaar), peter.hagoort@fcdonders.ru.nl (P. Hagoort).
}

For instance, sentence (1) is semantically reversible, because either of the two nouns is an equally probable candidate for the thematic role of agent. This is in contrast to sentence (2) that is semantically nonreversible, since bones cannot eat lions. While Broca patients usually have little difficulty with interpreting semantically nonreversible sentences, they often perform relatively poorly on reversible sentences (Berndt, Mitchum, \& Haendiges, 1996): whereas assignment of thematic roles (who was doing what to whom) seems to be constrained by real world knowledge in nonreversible sentences, these constraints are absent in the semantically reversible sentences.

The most prevalent means of testing the comprehension of semantically (non)reversible sentences has been the use of sentence-picture matching tasks. In this task, patients usually see a set of pictures. After some inspection time, a spoken sentence is presented. One of the pictures depicts the sentence content; the others are distracters. The patient's task is then to select from this set of pictures the one that best portrays the meaning of the presented sentence. By manipulating the 
kind of disagreement between the sentence and the distracter pictures, information can be obtained as to which aspects of the language system are affected in the patient. For instance, distracter pictures for semantically reversible sentences often portray a reversal of thematic roles of the sentence noun phrases (cf. Berndt et al., 1996).

During the last decades, the sentence-picture matching task has been applied to a variety of sentences of different structural types to probe Broca patients' ability to understand these types of sentences (Berndt, Mitchum, \& Wayland, 1997). This approach has revealed relatively good comprehension for canonical structures like actives and subject-relatives and poor comprehension for non-canonical structures like passives and object-relatives (Grodzinsky, Piñango, Zurif, \& Drai, 1999). Although there has been a lively debate regarding the proportion of Broca patients that actually manifest chance-level comprehension for non-canonical sentences (Berndt \& Caramazza, 1999; Berndt et al., 1996; Caramazza, Capitani, Rey, \& Berndt, 2001; Zurif \& Piñango, 1999), the tendency of Broca patients with agrammatic comprehension toward thematic reversals in the comprehension of semantically reversible passive sentences has been frequently observed.

A number of hypotheses have been offered to account for Broca patients' difficulty with knowing 'who is doing what to whom' in semantically reversible sentences (e.g. Grodzinsky, 1986, 1995, 2000; Haarmann, Just, \& Carpenter, 1997; Just \& Carpenter, 1992; Linebarger, 1990, 1995; Linebarger, Schwartz, \& Saffran, 1983; Miyake, Carpenter, \& Just, 1994, 1995; Schwartz, Linebarger, Saffran, \& Pate, 1987; Schwartz, Linebarger, \& Saffran, 1985). Most of these hypotheses have in common that they account for comprehension data that have been gathered almost exclusively from off-line tasks, especially the sentence-picture matching task. Such a task is off-line in the sense that patients are requested to operate on a final product of the language comprehension process (Hagoort \& Kutas, 1995). Usually, in sentence-picture matching tasks, subjects are asked to give their responses well after the sentence has been fully presented. The sentence-picture matching task in its classical form thus does not give any information on temporal aspects of the process of thematic role assignment that results in the overt performance on the task. In contrast to off-line tasks, on-line measures tap into the language comprehension process as it unfolds in real time. A measure that provides an on-line and continuous record of language related processing events is the registration of event-related brain potentials (ERPs). The present study will use ERPs to gain an insight into on-line thematic role assignment in patients with Broca's aphasia during sentence-picture matching.

\subsection{Present study}

The present study aimed to study on-line thematic role assignment in patients with Broca's aphasia during sentence-picture matching. ERPs were recorded while subjects were engaged in sentence-picture matching. They were presented with a picture on a computer screen. After some inspection time, the picture disappeared and a spoken sentence was presented over head- phones. After hearing the sentence, subjects pressed a button to indicate whether the sentence corresponded to the picture or not.

It is assumed here, that healthy subjects, when they are faced with a picture (e.g. a picture which portrays an action with the involvement of an agent and a recipient of the action) will form some kind of mental representation of the depicted event (Chatterjee, Maher, \& Heilman, 1995; Chatterjee, Southwood, \& Basilico, 1999). While the subjects hear the sentence, a linguistic representation of the sentence will be incrementally constructed as words come in. The match/non-match decision requires that this sentence representation is compared to the representation of the depicted event. In order to establish whether the sentences in our study correspond to the pictures or not, thematic role information is needed. As soon as this thematic role information is available, subjects can compare the roles described in the sentence to the roles depicted in the picture. The sentence will correspond to the picture if the thematic roles as expressed in the sentence are in agreement with the ones being portrayed. The sentence does not match the picture if thematic roles have been reversed.

The exact moments at which syntactic information in the context of visual information is used for thematic role assignment is still largely unknown. However, there are strong indications that, in real-time sentence comprehension, relevant visual context is immediately taken into account (Spivey, Tanenhaus, Eberhard, \& Sedivy, 2002; Tanenhaus, Spivey-Knowlton, Eberhard, \& Sedivy, 1995). With the on-line sentence picture matching paradigm the immediacy of thematic role assignment can be investigated. Consider the following example of a picture paired with a spoken sentence:

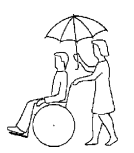

The tall man on this picture pushes the young woman

After presentation of a picture in which a woman pushes a man in a wheelchair, the sentence "The tall man on this picture pushes the young woman" is presented. The sentence part "The tall man pushes" allows the assignment of grammatical subject to man and, in combination with the active voice of the sentence, the mapping onto the thematic role of agent. However, there are two options for the moment at which the thematic role of patient can be assigned. One possibility is that, given the presence of a man and a woman on the picture, the thematic role of patient can already be assigned by inference to the woman, before the actual lexical item woman is heard. This immediacy option would imply that the mismatch between the event structure of the picture and the event structure of the sentence can already be detected upon hearing the verb form 'pushes', thus as soon as the argument structure belonging to the verb 'to push' is available and one of the thematic roles (i.e. the role of agent) has been assigned. An alternative option is that the assignment of the thematic role of patient waits for the actual lexical information that is to be associated with the thematic grid. This completeness option assumes that in the example above mismatch detection will emerge not until the word form woman is heard. Thus, if healthy control subjects assign thematic roles immediately, then 
the mismatch can be detected early in the sentence (i.e. at the verb); if subjects wait for full lexical information when assigning thematic roles, then the mismatch between the sentence and picture can only be detected after this information has been presented.

It is assumed that the detection of the mismatch between the sentence and the picture will be reflected in the electrophysiological signal (cf. Connolly \& D'Arcy, 2000; Connolly, D'Arcy, Newman, \& Kemps, 2000). For this purpose, the ERP profiles for matching versus non-matching sentences will be compared. This can indicate whether, and on the basis of which information the mismatch has been detected.

This study focuses on possible impairments in the on-line thematic role assignment in aphasia. We will compare the ERP signature of Broca's aphasics to the ERP signature observed in healthy controls for on-line thematic role assignment. Beforehand we do not make strong claims about which kind of ERP componentry will be involved. However, there are several possible candidates. For instance, as the detection of the (mis)match between the sentence and the picture requires some kind of semantic analysis, it is possible that an N400 effect may appear. Further, a P600 effect may be involved since, recently, Kuperberg, Sitnikova, Caplan, and Holcomb (2003) found such an effect when subjects were presented with sentences containing thematic role animacy violations. They interpreted this P600 as a result of a discrepancy between the probable thematic role and the actual assigned thematic role. In their view (but see also Kolk, Chwilla, van Herten, and Oor (2003)), this P600 could reflect an on-line attempt to structurally repair a sentence by reassigning thematic roles.

In order to be able to reliably interpret possible changes in the ERP effects of the Broca's aphasics as reflecting changes in their on-line language processing, it is important to identify factors that could contaminate the results of the experiment. To control for the non-specific effect of aging, the results of the Broca patients will be compared to a group of healthy age-matched controls. A group of non-aphasic patients with a lesion in the right hemisphere will be tested to control for non-specific effects of brain damage on the ERP effects (cf. Hagoort \& Kutas, 1995).

\section{Method}

\subsection{Subjects}

Ten patients with aphasia secondary to a single cerebral vascular accident (CVA) in the left hemisphere participated in this study. A group of fifteen healthy normal subjects, who were approximately matched in age and education level to the aphasic patients were tested to control for age and education effects. To account for non-specific effects of brain damage on cognitive ERP components, a group of eight non-aphasic patients with a single CVA in the right hemisphere (RH patients) was tested. All subjects gave informed consent, according to the declaration of Helsinki. The elderly control subjects and the RH patients were paid for their participation. The mean age of the aphasic patients was: 58.6 years (range: $44-73$ years), the RH patients were on average 61.6 years (range: 47-76 years) and the normal elderly controls had a mean age of 60.0 years (range: 52-74 years). All elderly control subjects were right-handed according to an abridged Dutch version of the Oldfield handedness inventory (Oldfield, 1971). Five of the elderly control subjects reported familial left-handedness. None of the elderly control subjects had any known neurological impairment or used neuroleptics, according to their responses on a questionnaire. The aphasic patients and the RH patients were premorbidly, all right-handed. All subjects had normal or corrected-to-normal vision. None of the subjects reported hearing loss.

All neurological patients were tested at least 21 months post-onset of their cerebral vascular accident. Median post-onset time for the patients with Broca's aphasia was 6.1 years (range: 1.9-11.1 years) and for the RH patients 3.3 years (range 2.2-7.3 years). All neurological patients were tested with the standardised Dutch version of the Aachen aphasia test (AAT) (Graetz, De Bleser, \& Willmes, 1992). Both presence and type of aphasia were diagnosed on the basis of the AAT results and on the basis of a transcribed sample of the patient's spontaneous speech. Three experts evaluated this spontaneous speech. All RH patients were diagnosed as non-aphasic and all left-hemisphere patients were diagnosed as patients with Broca's aphasia on the basis of a procedure that matches the individual score profiles against a norm population of patients. According to their scores on the comprehension subtest of the AAT, the aphasic patients had mild to moderate comprehension deficits. The presence of syntactic comprehension problems was determined by administering all subjects the Dutch version of an off-line test that assesses the influence of syntactic complexity on sentence comprehension (after Huber, Klingenberg, Poeck, \& Willmes, 1993). For this syntactic off-line test, participants listened to a total of 72 spoken sentences and selected one out of four pictures that matched each sentence. The test contained five levels of syntactic complexity, ranging from active, semantically irreversible sentences (e.g. "The man with the tie carries the ball") to sentences containing an embedded subject-relative clause in the passive voice (e.g. "The child that is sought by the man carries a ball"). Statistical evaluation of the syntactic off-line test results (see Table 1) confirms the syntactic comprehension problems of the Broca patients as compared to the normal controls and the RH control patients. The pattern of results substantiates the syntactic comprehensions problems of the Broca patients in this study. All patients were also tested with the subtest sentence comprehension of the WEZT (Werkwoorden- en Zinnen test) (Bastiaanse, Maas, \& Rispens, 2000). This subtest assesses whether a patient is able to attribute correct thematic roles to different sentence constituents. Broca patients performed relatively poorly when compared to the RH patients. Based on the patients' scores on the token test, which is a valid measure of the general severity of the aphasia (Orgass, 1986), the general severity of the aphasia ranged from light to severe. For further patient information, see Table 1 .

\subsection{Materials}

The stimuli for this experiment consisted of a list of 316 pairs of a picture and a spoken sentence. Of these picture-sentence pairs, 288 were the critical pairs for the experiment. The remaining pairs were used as practice trials (12) and filler items (16). Pictures (96) came from a German syntax test (Huber et al., 1993) and consisted of simple black on white line drawings. Half of these pictures depicted semantically irreversible situations (e.g. a woman is reading a book), half depicted semantically reversible situations (e.g. a man is pushing a woman in a wheel chair). Sentences were taken from the Dutch adaptation of this German syntax test (Ter Keurs, Brown, Hagoort, \& Stegeman, 1999; Wassenaar \& Hagoort, 1994). The sentences were slightly adapted for the purposes of this experiment and were paired with the pictures. Three different sentence types were combined with matching/non-matching pictures in a full factorial design (see Fig. 1). The sentence types were: (I) semantically irreversible active sentences; (II) semantically reversible active sentences; (III) semantically reversible passive sentences. With these sentences we could vary (1) semantic (ir)reversibility and (2) syntactic complexity. It has often been reported that Broca patients have typically more problems with reversible than irreversible sentences. Contrary to irreversible sentences, reversible sentences provide no lexical-semantic but only syntactic cues to assign thematic roles. In addition, passive reversible sentences are for Broca patients usually more difficult to understand than their active counterparts (but see Berndt et al., 1996). We therefore included these different sentence types to see whether the process of on-line thematic role assignment, as revealed in the electrophysiological profile, was differentially affected by semantic (ir)reversibility and syntactic complexity.

A sentence matched a picture if the sentence content was in agreement with the depicted event. Consequently, if the sentence content did not correspond to the depicted event, the sentence and the picture did not match. 
Table 1

Individual patient information for the patients with Broca's aphasia and the non-aphasic RH patients

\begin{tabular}{|c|c|c|c|c|c|c|c|}
\hline Patient & Age & Sex & Token test ${ }^{\mathrm{a}}$ & $\begin{array}{l}\text { Overall compreh. } \\
\text { score AAT }\end{array}$ & $\begin{array}{l}\text { Syntactic off-line } \\
\text { score }^{c}\end{array}$ & $\begin{array}{l}\text { Sentence compreh. } \\
\text { WEZT }^{\text {d }}\end{array}$ & Lesion site \\
\hline 1. Broca & 55 & $\mathrm{~F}$ & 10 & $97 / 120$ & $95 / 144$ & $30 / 40$ & Left fronto-temporo-parietal incl. insula \\
\hline 2. Broca & 70 & $\mathrm{~F}$ & 11 & $103 / 120$ & $111 / 144$ & $31 / 40$ & No adequate $\mathrm{CT}$ information available \\
\hline 3. Broca & 60 & M & 9 & $111 / 120$ & $90 / 144$ & $27 / 40$ & Left capsula interna \\
\hline 4. Broca & 48 & M & 17 & $94 / 120$ & $93 / 144$ & $37 / 40$ & Left temporo-parietal \\
\hline 5. Broca & 51 & $\mathrm{~F}$ & 21 & $84 / 120$ & $104 / 144$ & $27 / 40$ & Left parieto-occipital \\
\hline 6. Broca & 53 & M & 42 & $89 / 120$ & $74 / 144$ & $21 / 40$ & No adequate CT information available \\
\hline 7. Broca & 69 & $\mathrm{~F}$ & 18 & $91 / 120$ & $51 / 144$ & $34 / 40$ & Left fronto-temporal incl. insula \\
\hline 8. Broca & 73 & M & 18 & $89 / 120$ & $60 / 144$ & $23 / 40$ & Left temporal \\
\hline 9. Broca & 63 & M & 46 & $86 / 120$ & $106 / 144$ & $20 / 40$ & Left fronto-temporal \\
\hline 10. Broca & 44 & $\mathrm{~F}$ & 14 & $94 / 120$ & $88 / 144$ & $32 / 40$ & Left parieto-occipital \\
\hline 1. $\mathrm{RH}$ & 52 & M & 0 & $113 / 120$ & $134 / 144$ & $40 / 40$ & No adequate $\mathrm{CT}$ information available \\
\hline 2. $\mathrm{RH}$ & 56 & M & 9 & $106 / 120$ & $128 / 144$ & $38 / 40$ & Right insular \\
\hline 3. RH & 67 & M & 2 & $116 / 120$ & $137 / 144$ & $40 / 40$ & Right parietal \\
\hline 4. RH & 69 & $\mathrm{~F}$ & 2 & $108 / 120$ & $135 / 144$ & $39 / 40$ & Right basal ganglia \\
\hline 5. $\mathrm{RH}$ & 66 & $\mathrm{~F}$ & 1 & $120 / 120$ & $143 / 144$ & $40 / 40$ & Right temporo-parietal \\
\hline 6. RH & 76 & M & 0 & $102 / 120$ & $139 / 144$ & $40 / 40$ & Right parietal \\
\hline 7. $\mathrm{RH}$ & 60 & $\mathrm{~F}$ & 1 & $103 / 120$ & $125 / 144$ & $40 / 40$ & Right basal ganglia \\
\hline 8. RH & 47 & $\mathrm{~F}$ & 0 & $108 / 120$ & $126 / 144$ & $37 / 40$ & Right fronto-temporal \\
\hline
\end{tabular}

${ }^{a}$ Severity of the aphasic disorder as indicated by the token test: no/very mild disorder (0-6); light (7-23); middle (24-40); severe (41-50). RH patient 2 had a token test score of 9, but was, on the basis of his spontaneous speech and ALLOC classification (a procedure that matches individual score profiles against a norm population of patients) non-aphasic.

${ }^{\mathrm{b}}$ Severity of the comprehension disorder as indicated by the Aachen aphasia test subtest on comprehension (includes word and sentence comprehension in both the auditory and visual modality): no/very mild disorder (107-120); light (90-106); middle (67-89); severe (1-66). Compreh.: comprehension; AAT: Aachen aphasia test.

${ }^{c}$ Range of performance in healthy control subjects is 132-144. ANOVAs on the percentage-correct scores of the sentences with increasing syntactic complexity of the off-line test showed that syntactic complexity had a differential effect on the comprehension scores of the different subject groups [complexity: $F$ (3.83, $114.75)=35.58$, M.S.E. $=79.77, p=0.000$; Group: $F(2,30)=36.52$, M.S.E. $=514.24$; complexity $\times$ group: $F(7.65,114.75)=12.36$, M.S.E. $=79.77, p=0.000]$. Post hoc analyses $(\alpha=0.05)$ revealed that the Broca patients performed significantly worse than both the normal controls and the RH controls. The two control groups did not differ significantly from each other.

d The subtest sentence comprehension of the WEZT assesses whether a patient is able to attribute the correct thematic roles to different sentence constituents. Range of performance in healthy subjects is 39-40 (Bastiaanse et al., 2000).

For the semantically irreversible sentences (sentence type I), identical pictures $(N=48)$ were used in the matching and non-matching condition, but the sentences were different (see Fig. 1a). A sentence mismatched the picture when the patient role from the picture was placed into the grammatical subject position of the sentence.

For the semantically reversible sentences (sentence types II and III) identical sentences were used in the matching and non-matching condition, but the pictures were different (see Fig. $1 \mathrm{~b}$ and c). A sentence did not match the picture when the thematic roles of agent and patient had been reversed. For all sentence type conditions, each picture was presented twice and was paired with a matching sentence and a mismatching sentence, respectively. This resulted for each sentence type in a total of $96(48 \times 2)$ experimental picture-sentence pairs. The list of experimental items is available upon request.

It has been reported that perceptual characteristics of pictures (like relative position of agent/patient, the direction of an action) affect the ease of both production and comprehension of sentences (e.g. Chatterjee et al., 1999; Flores D'Arcais, 1973). Therefore, the factors of position of agent/patient and the direction of action have been carefully counterbalanced.

A female speaker spoke all the experimental sentences, the fillers and the practice sentences, at a normal speaking rate. Sentences were spoken in a sound attenuating booth and recorded on a digital audiotape. The stimuli were stored on a hard disk. A speech waveform editing system (Xwaves/ESPS package) was used to mark the onset and offset of each sentence, together with the onset of various words within the sentences. Sentence length for the active and passive sentences was 10 and 11 words, respectively. Mean sentence duration was $2734 \mathrm{~ms}$ (S.D. $188.1 \mathrm{~ms}$ ) for the active, semantically irreversible sentences, $2587 \mathrm{~ms}$ (S.D. $217.4 \mathrm{~ms}$ ) for the active, semantically reversible sentences, and $2712 \mathrm{~ms}$ (S.D. $206.1 \mathrm{~ms}$ ) for the passive semantically reversible sentences.
On the basis of these materials two experimental lists were created. Approximately, an equal number of subjects was assigned to each list. For the first list, all the experimental picture-sentence pairs were distributed over four blocks such that a matching and a non-matching pair were never presented in the same block. Within each block the items were presented in a pseudo-randomised order with the constraint that there were never more than three successive trials with the same sentence type. Repeated pictures were always separated by at least three other items. Successive matching or non-matching trials never occurred more than three times in a row. The second list was derived from the first by reversing the presentation order of the blocks. Each experimental list was preceded by a practice list of 12 picture-sentence pairs. A number of 16 filler trials were added to each list (four per block).

\subsection{Procedure}

Participants were tested individually in a dimly illuminated soundattenuating booth. They were seated in a comfortable reclining chair (apart from five patients who had to be tested in their wheelchair), in front of a computer screen. Viewing distance was approximately $100 \mathrm{~cm}$. A trial started with the presentation of a picture for $4 \mathrm{~s}$. After picture offset, a fixation point (i.e. an asterisk) appeared, to warn the subjects that they had to fixate their eyes on the middle of the screen. At $1000 \mathrm{~ms}$ after the onset of the asterisk, a sentence was presented. Participants were asked not to blink or to move their eyes during the period in which the fixation point was on the screen. The fixation point remained on the screen until $1000 \mathrm{~ms}$ after sentence offset and was followed by a horizontal rectangle. The appearance of the rectangle signalled that a judgement task had to be carried out: if the sentence matched the picture, subjects were instructed to press the green button on a response 


\begin{tabular}{|l|l|}
\hline Picture & $\begin{array}{l}\text { Sentence type I: Semantically irreversible active sentences } \\
\text { De jonge vrouw op dit plaatje leest het spannende boek } \\
\text { (The young woman on this picture reads the exciting book) }\end{array}$ \\
(The exciting book on this picture reads the young woman)
\end{tabular}

(a)

\begin{tabular}{|l|l|}
\hline Picture & Sentence type II: Semantically reversible active sentences \\
(The tall man on this picture pushes the young woman) \\
Dismatch: De lange man op dit plaatje duwt de jonge vrouw \\
(The tall man on this picture pushes the young woman)
\end{tabular}

(b)

\begin{tabular}{|l|l|}
\hline Picture & Sentence type III: Semantically reversible passive sentences \\
(The woman on this picture is pushed by the tall man) \\
De vrouw op dit plaatje wordt geduwd door de lange man \\
(The woman on this picture is pushed by the tall man)
\end{tabular}

(c)

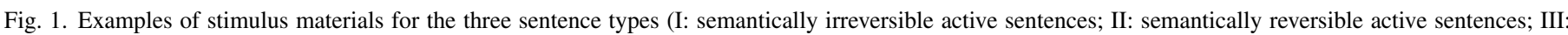

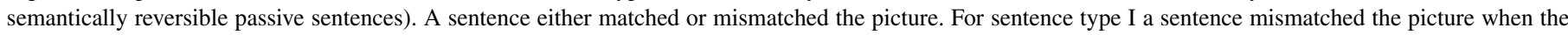

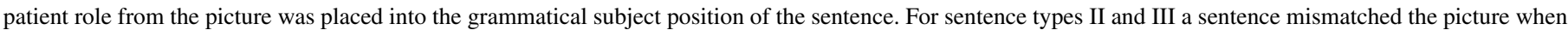
the thematic roles of agent and patient had been reversed.

button box which was placed in front of them. If the sentence did not match the picture, the red button had to be pressed. We used this delayed judgement task to eliminate effects of motor response preparation on the ERPs of interest. The position of the green and red button on the button box (i.e. left and right) was counterbalanced across subjects. Due to the occurrence of hemiparesis in a number of patients, Broca patients were required to respond with their left index finger, and the RH patients with their right index finger. Half of the normal control subjects had to press with their left index finger, half of them with their right index finger. Push button responses and latencies were measured from sentence offset. The horizontal rectangle disappeared as soon as the response button was pressed. The time-out period (the moment in time after which responses were registered as missing) was set at $4000 \mathrm{~ms}$. Two seconds after either pressing the push button or passing the time-out period, a new trial started.

The pictures were presented on a computer screen, in black on a white background. The presentation of the pictures and the sentences, and the acquisition of the response button data were controlled by NESU, a stimulus presentation system developed at the Max Planck Institute for Psycholinguistics. The subjects listened to the stimuli via a closed-ear Sennheiser HMD-224 headphone.

At the beginning of a session, participants were given a booklet containing all experimental pictures. They were asked to carefully look at the pictures. When a participant indicated that he/she had looked at all the pictures, the practice block was presented to familiarize the subjects with the task. The actual experiment consisted of four experimental blocks of approximately 16 min each. Subjects were given short breaks between the blocks.

\subsection{EEG-recording}

Continuous EEG was recorded from $29 \mathrm{Ag} / \mathrm{AgCl}$-sintered electrodes mounted in an elastic cap, each referred to the left mastoid. Twenty-three electrodes (Fz, FCz, Cz, Pz, Oz, AF3, AF4, F7, F8, F3, F4, FT7, FT8, FC3, $\mathrm{FC} 4, \mathrm{C} 3, \mathrm{C} 4, \mathrm{CP} 3, \mathrm{CP} 4, \mathrm{P} 3, \mathrm{P} 4, \mathrm{PO} 7, \mathrm{PO} 8)$ were placed according to the standard system of the American Electroencephalographic Society (1994). Six electrodes were placed over non-standard intermediate locations: (a) a temporal pair (LT and RT) placed laterally to $\mathrm{Cz}$, at $33 \%$ of the interaural distance, (b) a temporo-parietal pair (LTP and RTP) placed $30 \%$ of the interaural distance lateral and $13 \%$ of the nasion-inion distance posterior to $\mathrm{Cz}$, and (c) a parietal pair midway between LTP/RTP and PO7/PO8 (LP and RP). Vertical eye movements were monitored via a supra- to suborbital bipolar montage. A right to left canthal bipolar montage was used to monitor for horizontal eye movements. Activity over the right mastoid bone was recorded on an additional channel to determine if there were differential contributions of the experimental variables to the presumably neutral mastoid site. No such differential effects were observed. The EEG and EOG recordings were amplified by a SynAmp ${ }^{\mathrm{TM}}$ Model 5083 EEG amplifier system (Neuroscan) using a band-pass filter of $0.05-30 \mathrm{~Hz}$. Impedances were kept below $3 \mathrm{k} \Omega$. The 
EEG and EOG signals were digitised on-line with a sampling frequency of $200 \mathrm{~Hz}$.

\section{Results}

\subsection{Behavioral results}

\subsubsection{Data analysis}

The results for the normal control subjects and the neurological patients were analysed separately. Analyses were done on response latency data and on error data. Response latencies were measured from sentence offset to the onset of the reaction of the participant. Analyses of the latency data were based on the subjects' median response latency in each condition. Errors and missing values (due to time-outs) were replaced for every subject by his/her median per condition. Analyses of the error data were done on the number of errors per participant per condition. Repeated Measures Analyses of Variance were performed with sentence type (three levels: semantically irreversible active, semantically reversible active, and semantically reversible passive) and matching (two levels: matching, nonmatching) as within-subjects factors. A Huynh-Feldt correction was applied to all repeated measures with more than one degree of freedom in the numerator. The Tukey-HSD multiple range test $(\alpha=0.05)$ was used for post hoc analysis.

\subsubsection{Latency analyses}

The results for the normal control subjects and both patient groups are summarized in Fig. 2. Inspection of this figure reveals

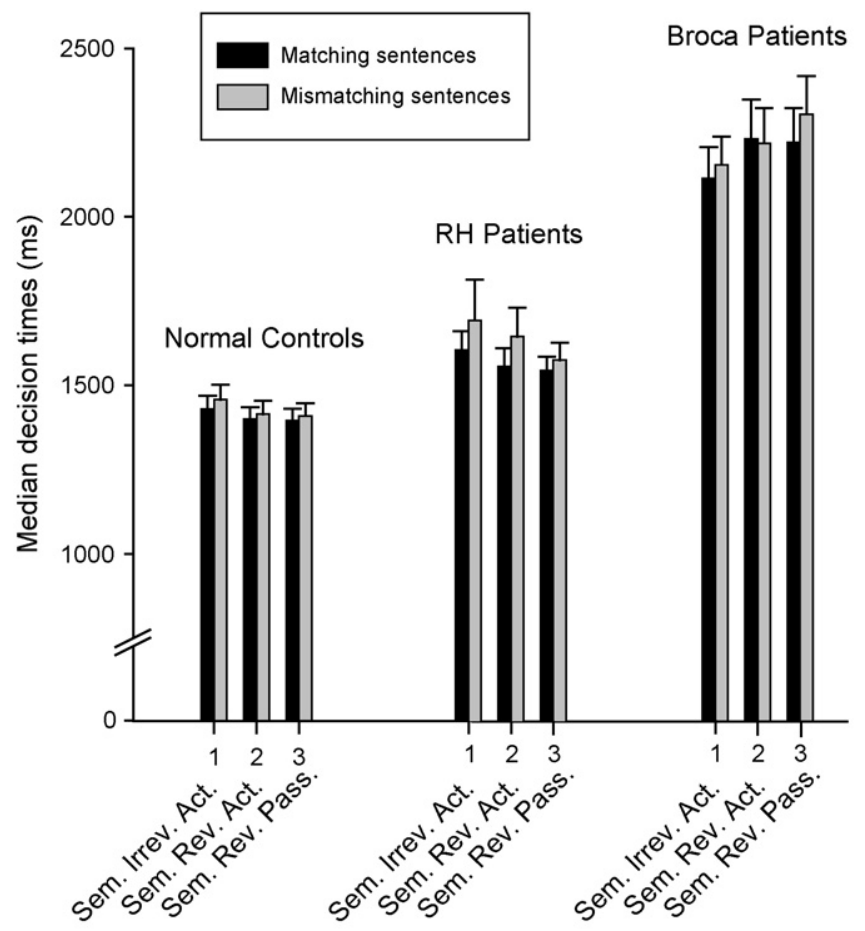

Fig. 2. Mean decision times (ms) as a function of Sentence and matching type for the group of normal controls $(N=15), \mathrm{RH}$ patients $(N=8)$, and Broca patients $(N=10)$. Average percentage of time-outs was 1.02 for normal controls, 1.3 for $\mathrm{RH}$ patients, and 3.4 for Broca patients. that the quickest responses were given by the normal control subjects. The RH patients performed somewhat slower than the normal controls, whereas the by far longest decision times came from the Broca patients.

For the normal control subjects, in the overall ANOVA, there was a main effect of sentence type $(F(1.4,19.2)=17.9$; $p=0.000)$. There was no significant effect of matching $(F(1$, $14)=1.71 ; p=0.212$ ), nor a significant sentence type by matching interaction $(F<1)$. Post hoc comparisons revealed that normal control subjects responded significantly more slowly to the irreversible active sentences $\left(\mathrm{RT}_{\text {irrev act }}=1443 \mathrm{~ms}\right)$ than to both types of reversible sentences $\left(\mathrm{RT}_{\mathrm{rev} \text { act }}=1406 \mathrm{~ms}\right.$ and $\mathrm{RT}_{\text {rev pass }}=1401 \mathrm{~ms}$, respectively).

For the $R H$ patients, the overall ANOVA did not yield any significant effect [sentence type: $F(1.4,9.5)=2.38 ; p=0.152$; matching: $F(1,7)=2.00 ; p=0.20$; sentence type $\times$ matching: $F<1]$.

Whereas both normal control subjects and RH patients responded slowest to the irreversible active sentences, the overall data of the Broca patients showed just an increase in response latency from the irreversible active sentences to the reversible passive sentences. In the overall ANOVA, there was no main effect of sentence type, but a tendency for response latency to be longer in the reversible passive sentences $(F(1.34,12.08)=3.53$; $p=0.076)$. There was no significant effect of matching $(F<1)$. Also the sentence type $\times$ matching interaction did not reach significance level $(F(2,18)=2.36 ; p=0.123)$.

An analysis with group of subjects as additional betweensubjects factor revealed a significant group of subjects effect $(F(2,30)=42.74 ; p=0.000)$. A post-hoc analysis showed that the Broca patients overall responded significantly slower than both the normal controls and the $\mathrm{RH}$ patients. The overall response latencies of the normal controls and $\mathrm{RH}$ patients did not differ significantly. In addition, there was a significant group of subjects by sentence type interaction $(F(3.01,45.11)=6.16$; $p=0.001)$. This interaction becomes understandable if one takes a look at Fig. 2. As visualized in this figure, both normal controls and $\mathrm{RH}$ patients show decreasing decision times over sentence types, whereas the Broca patients show increasing decision times.

\subsubsection{Error analyses}

Fig. 3 summarizes the error data for both normal control subjects and patient groups. As can be seen from this figure, the highest error percentages were found for the Broca patients, whereas RH patients and normal control subjects showed relatively low error percentages.

The normal control subjects made errors on $2.8 \%$ of the critical sentences, without significant differences between conditions [sentence type: $F<1$; matching: $F(1,14)=1.09 ; p=0.315$; sentence type $\times$ matching: $F(1.02,14.21)=1.29 ; p=0.276)$ ].

The group of $\mathrm{RH}$ patients made errors on $3.1 \%$ of the sentences. No condition effects were obtained [sentence type: $F(1.75,12.23)=2.31 ; p=0.145$; matching: $F(1,7)=3.04$; $p=0.125$; sentence type $\times$ matching: $F<1]$.

The group of Broca patients had an overall error score of $23.8 \%$. Although this error percentage is substantial, it should be 


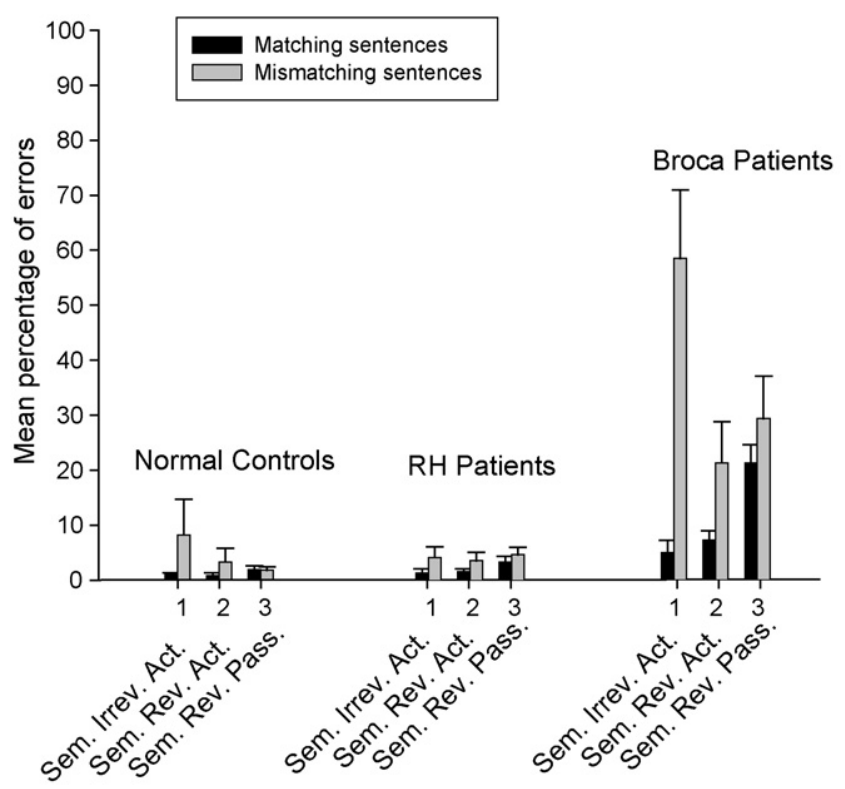

Fig. 3. Mean percentage of errors as a function of sentence and matching type for the group of normal controls $(N=15)$, RH patients $(N=8)$, and Broca patients $(N=10)$.

pointed out that these patients still performed above chance, with the exception of the non-matching irreversible active sentences. The large number of errors for the matching passive sentences suggests a tendency in the patients to interpret the passives as active sentences. Statistical analysis showed the following. A significant main effect emerged for sentence type $(F(1.53$, $13.80)=5.43 ; p=0.024)$ and also for matching $(F(1,9)=15.37$; $p=0.004)$. These main effects were qualified by a significant sentence type $\times$ matching interaction $(F(1.08,9.75)=6.39$; $p=0.029)$ : as can be seen in Fig. 3, the matching effect was disproportionately large for sentence type $1(F(1,9)=14.81$; $p=0.004)$. Furthermore, significantly more errors were made for the matching passive sentences than for the other matching sentences $(F(1.68,15.15)=12.14 ; p=0.001)$.

An analysis with group of subjects as additional betweensubjects factor revealed a significant group of subjects effect $(F(2,30)=28.13 ; p=0.000)$. A post hoc analysis showed that the Broca patients made overall significantly more errors than both the normal controls and the RH patients. The overall error rates of the normal controls and $\mathrm{RH}$ patients did not differ significantly from each other. In addition, there were significant interactions between group of subjects and sentence type $(F(2.90,43.52)=3.84 ; p=0.017)$, group of subjects and matching $(F(2,30)=9.13 ; p=0.001)$ and there was a significant threeway interaction of group of subjects $\times$ sentence type $\times$ matching $(F(2.32,34.84)=4.69 ; p=0.012)$. All these interactions could be attributed to the response pattern of the Broca patients, since all interactions disappeared when a between-subjects analysis was carried out in which the Broca patients were left out.

In conclusion, the normal control subjects responded slower to the semantically irreversible sentences than to the other sentence conditions, but their error rates did not differ between conditions. For the RH patients the different sentence types

had no differential influence on either response latency or error rates. The Broca patients showed, overall, much longer response latencies than both other subject groups. Also their overall error percentage was much higher, with the highest error rates for the non-matching irreversible active sentences.

\subsection{EEG-experiment}

Prior to off-line averaging, all single trials waveforms were screened for electrode drifting, amplifier blocking, muscle artefacts, eye movements and blinks. This was done over an epoch that ranged from $150 \mathrm{~ms}$ before to $3770 \mathrm{~ms}$ (=longest sentence plus $500 \mathrm{~ms}$ ). Trials containing artefacts were rejected. However, for subjects with a substantial number of blinks, single trials were corrected via a procedure described by Gratton, Coles, and Donchin (1983). This correction procedure removes the contribution of eye blinks from the ERP recorded at each electrode site. After artefact rejection, the overall rejection rate was $8.8 \%$ for the normal elderly controls, $15.4 \%$ for the $\mathrm{RH}$ patients, and $13.2 \%$ for the patients with Broca's aphasia. For all groups, rejected trials were evenly distributed among conditions. For each subject, average waveforms were computed across all remaining trials per condition after normalizing the waveforms of the individual trials on the basis of a $150 \mathrm{~ms}$ prestimulus baseline.

Several latency windows were selected for statistical analysis (see Table 2). These latency windows were determined after careful visual inspection of the waveforms and depended upon the time interval in which maximal differences between conditions were obtained. If necessary also additional latency ranges were analysed.

Subsequent ANOVAs used mean amplitude values computed for each subject, condition and electrode site in the selected latency windows. For each subject group, the results were first analysed in an omnibus ANOVA that crossed matching with the 29-level electrode factor. Scalp distributions were subsequently explored in an ANOVA with Site (four quadrants: anterior left (AL: AF3, F3, F7, FC3, FT7), anterior right (AR: AF4, F4, F8, FC4, FT8), posterior left (PL: CP3, LTP, P3, LP, PO7), and posterior right (PR: CP4, RTP, P4, RP, PO8)) as within-subjects

Table 2

Latency windows that were selected for statistical analysis

Semantically irreversible active sentences

Semantically reversible active sentences

Semantically reversible passive sentences
100-200 ms following acoustic onset verb 500-800 ms following acoustic onset verb 250-600 ms following acoustic onset sentence-final noun

150-300 ms following acoustic onset verb 500-700 ms following acoustic onset verb 250-600 ms following acoustic onset sentence-final noun

250-350 ms following acoustic onset auxiliary verb 450-700 ms following acoustic onset auxiliary verb 400-700 ms following acoustic onset sentence-final noun 
factor. The Huyhn-Feldt correction was applied when evaluating effects with more than one degree of freedom in the numerator, to compensate for inhomogeneous variances and co-variances across treatment-levels. The adjusted degrees of freedom and $p$-values will be presented. To test for differences between the results for the normal elderly control subjects and the patient groups, also group analyses are performed in the specified timewindows, with matching as within-subject factor and group of subjects as between-subjects factor.

In the following, first all the ERP data of the normal control subjects for the different experimental conditions will be presented, followed by the data of the RH patients, and finally, the data of the Broca patients will be shown.

\subsection{Normal control subjects}

\subsubsection{Semantically irreversible active sentences}

Fig. 4 displays for the semantically irreversible active sentences grand average waveforms for two representative electrodes and isopotential voltage maps, time-locked to the acoustic onset of the critical verb (upper panel) and sentencefinal noun (lower panel). Table 3 shows the relevant statistical results for the different time epochs, belonging to this condition. Waveforms were time-locked to the onset of the verb for the following reason. It is the verb that provides the argument structure of the sentence. This implies that the earliest possible moment in the sentence at which a mismatch between picture and
(A) Semantically irreversible (active)
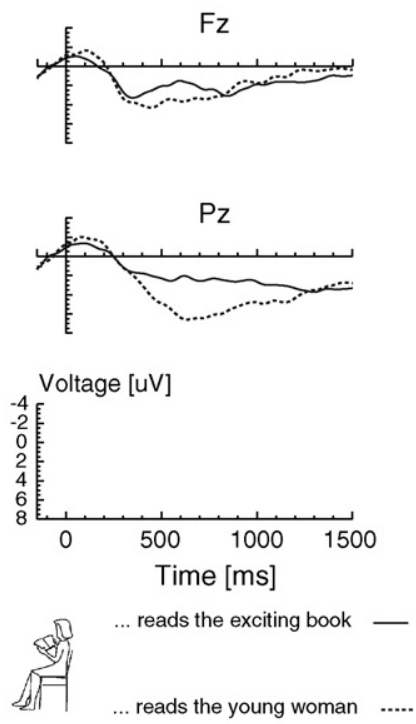

(B)
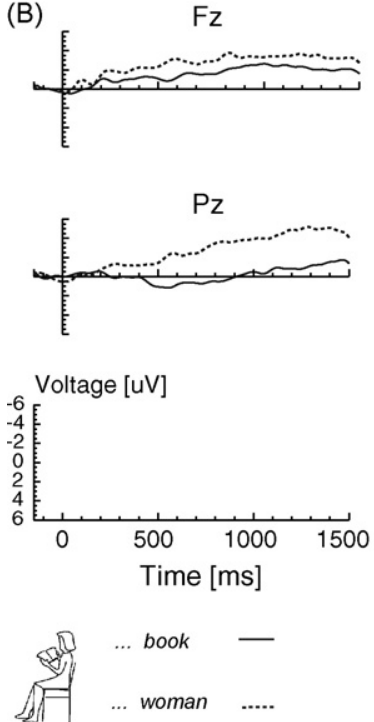

Normal Controls $(N=15)$

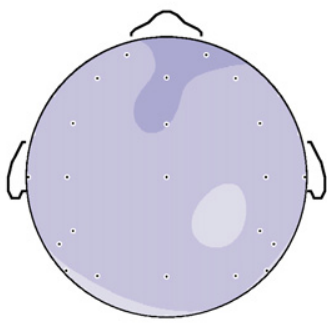

$100-200 \mathrm{~ms}$

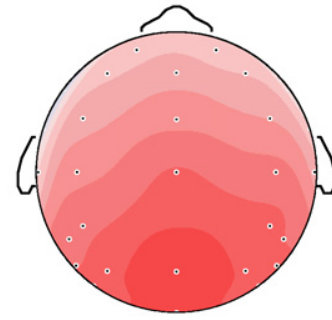

$500-800 \mathrm{~ms}$
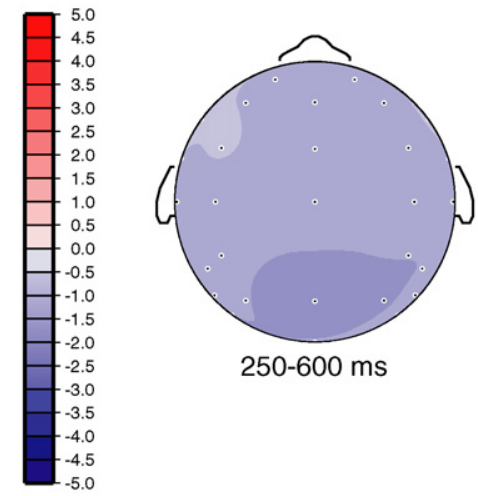

$250-600 \mathrm{~ms}$

Fig. 4. Grand average waveforms for two representative electrodes (Fz, Pz) and isopotential voltage maps, time-locked to the acoustic onset of the verb (A) and sentence-final noun (B) for the normal control subjects $(N=15)$ for the semantically irreversible active sentences. Dotted line is used for the mismatching sentences, solid line for the matching counterparts. Isopotential voltage maps were based on mean amplitude differences in the specified latency windows. Positive polarity effects are in red, negative-going effects are in blue. Scale values are in $\mu \mathrm{V}$. (For interpretation of the references to colour in this figure legend, the reader is referred to the web version of the article.) 
Table 3

Semantically irreversible active sentences for normal control subjects: mean ERP amplitude ANOVAs in the specified latency ranges

\begin{tabular}{|c|c|c|c|c|}
\hline Time-locking & Source & d.f. & $F$ & $p$ \\
\hline \multirow{2}{*}{$100-200 \mathrm{~ms}$ following acoustic onset verb } & Omnibus ANOVA (Mat) & 1,14 & 7.65 & $0.015^{*}$ \\
\hline & Quadrant ANOVA (Mat $\times$ site) & $2.47,34.59$ & 0.61 & 0.585 \\
\hline \multirow{4}{*}{$500-800 \mathrm{~ms}$ following acoustic onset verb } & Omnibus ANOVA (Mat) & 1,14 & 7.45 & $0.015^{*}$ \\
\hline & Quadrant ANOVA (Mat $\times$ site) & $2.65,37.04$ & 24.48 & $0.000^{* *}$ \\
\hline & Ant. vs. Post. ANOVA $($ Mat $\times \mathrm{A} / \mathrm{P})$ & 1,14 & 52.04 & $0.000^{* *}$ \\
\hline & Posterior ANOVA (Mat) & 1,14 & 15.92 & $0.001^{* *}$ \\
\hline \multirow{3}{*}{$250-600 \mathrm{~ms}$ following acoustic onset sentence-final noun } & Omnibus ANOVA (Mat) & 1,14 & 8.51 & $0.011^{*}$ \\
\hline & Quadrant ANOVA (Mat $\times$ site) & $1.85,25.94$ & 4.22 & $0.028^{*}$ \\
\hline & Ant. left vs. Post. left (Mat $\times$ AL/PL) & 1,14 & 5.37 & $0.036^{*}$ \\
\hline
\end{tabular}

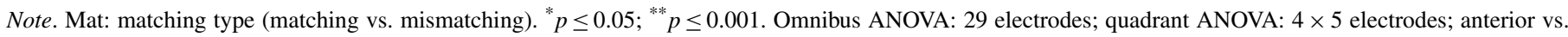

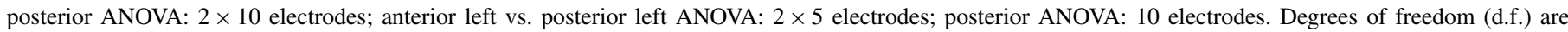
Huynh-Feldt corrected.

sentence could be detected is at the verb's position. A different option is that listeners wait until all argument positions are filled with lexical information. Waveforms were also timelocked to the sentence-final noun, since often ERP waveforms show global processing consequences downstream from local processing problems earlier in the sentence (Hagoort, 2003; Hagoort, Brown, \& Groothusen, 1993; Osterhout \& Holcomb, 1992, 1993).

As can be seen in Fig. 4A, in an early time epoch between approximately 50 and $250 \mathrm{~ms}$, a small negativity is elicited by the critical verb in the mismatching condition (see Fig. 4A). This negative effect is followed by a positive shift with a predominant centro-posterior distribution. The omnibus ANOVA for the 100-200 ms window focusing on the negative effect resulted in a significant effect of matching (see Table 3). Also the positive shift tested in the 500-800 ms latency window was significant, and it was significantly larger over posterior than anterior regions (see Table 3).

The sentence-final noun (see Fig. 4B) elicited a broad and sustained negative shift in the mismatching condition, starting at $200 \mathrm{~ms}$ post-onset and continuing throughout the remaining epoch. This effect was significant (see Table 3) and largest over posterior sites.

\subsubsection{Semantically reversible active sentences}

Fig. 5 displays for the semantically reversible active sentences grand average waveforms for two representative elec- trodes and isopotential voltage maps, time-locked to the acoustic onset of the critical verb (upper panel) and sentence-final noun (lower panel). Table 4 shows the relevant statistical results for the different time epochs, belonging to this condition.

The critical verb in the mismatching condition elicited at centro-posterior sites a marked negative-going wave in the $50-450 \mathrm{~ms}$ epoch, for anterior sites between approximately 50 and $300 \mathrm{~ms}$ (see Fig. 5A). The negativity was significant (see Table 4) and largest over posterior sites. The negative effect was followed by a positive shift. For the anterior sites the positivity started at about $300 \mathrm{~ms}$ extending up to $700 \mathrm{~ms}$. At posterior sites this positive effect started somewhat later (at around $500 \mathrm{~ms}$ ). However, the positivity failed to reach significance (see Table 4).

The sentence-final noun elicited in the mismatching condition a broad, widely distributed sustained negative shift (see Fig. 5B). This effect was significant (see Table 4).

\subsubsection{Semantically reversible passive sentences}

Fig. 6 displays grand average waveforms for two representative electrodes and isopotential voltage maps, time-locked to the acoustic onset of the auxiliary verb (upper panel) and sentencefinal noun (lower panel). For this condition, the auxiliary verb (instead of the verb) was used as critical time-locking point. Although it is the main verb that provides the argument structure, it is not unconceivable that, in the context of visual information, which provides constraints on possible arguments, the information of the auxiliary verb already is used, to detect a (mis)match

Table 4

Semantically reversible active sentences for normal control subjects: mean ERP amplitude ANOVAs in the specified latency ranges

\begin{tabular}{|c|c|c|c|c|}
\hline Time-locking & Source & d.f. & $F$ & $p$ \\
\hline \multirow{4}{*}{$150-300 \mathrm{~ms}$ following acoustic onset verb } & Omnibus ANOVA (Mat) & 1,14 & 7.54 & $0.016^{*}$ \\
\hline & Quadrant ANOVA (Mat × site) & $2.57,35.94$ & 7.27 & $0.001^{* *}$ \\
\hline & Ant. vs. Post. ANOVA $($ Mat $\times \mathrm{A} / \mathrm{P})$ & 1,14 & 13.65 & $0.002^{*}$ \\
\hline & Posterior ANOVA (Mat) & 1,14 & 12.79 & $0.003^{*}$ \\
\hline \multirow{2}{*}{$500-700 \mathrm{~ms}$ following acoustic onset verb } & Omnibus ANOVA (Mat) & 1,14 & 2.21 & 0.159 \\
\hline & Quadrant ANOVA (Mat × site) & $2.36,33.09$ & 0.58 & 0.591 \\
\hline \multirow{2}{*}{$250-600 \mathrm{~ms}$ following acoustic onset sentence-final noun } & Omnibus ANOVA (Mat) & 1,14 & 7.65 & $0.015^{*}$ \\
\hline & Quadrant ANOVA (Mat × site) & $1.99,27.81$ & 0.31 & 0.732 \\
\hline
\end{tabular}

Note. Mat: matching type (matching vs. mismatching). ${ }^{*} p \leq 0.05 ;{ }^{* *} p \leq 0.001$. Omnibus ANOVA: 29 electrodes; quadrant ANOVA: $4 \times 5$ electrodes; anterior vs. posterior ANOVA: $2 \times 10$ electrodes; posterior ANOVA: 10 electrodes. Degrees of freedom (d.f.) are Huynh-Feldt corrected. 
(A) Semantically reversible (active)
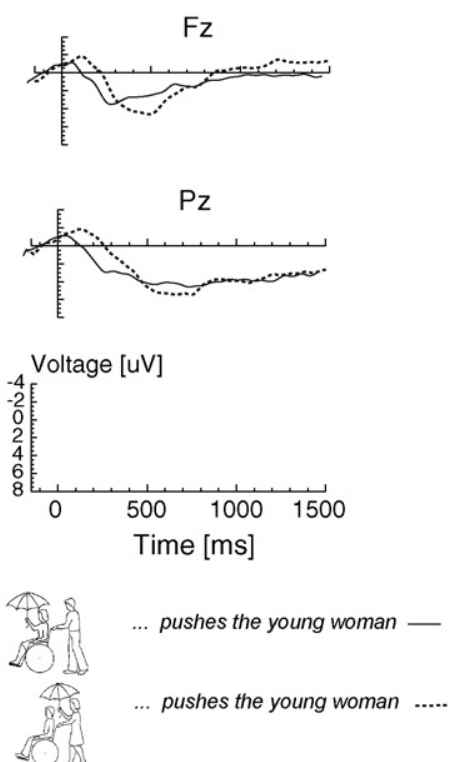

(B)
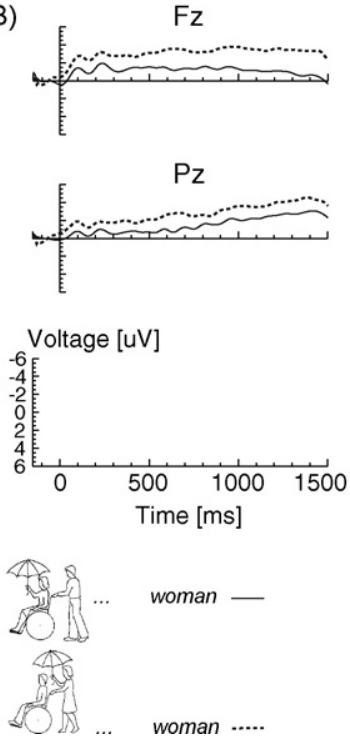

Normal Controls $(N=15)$
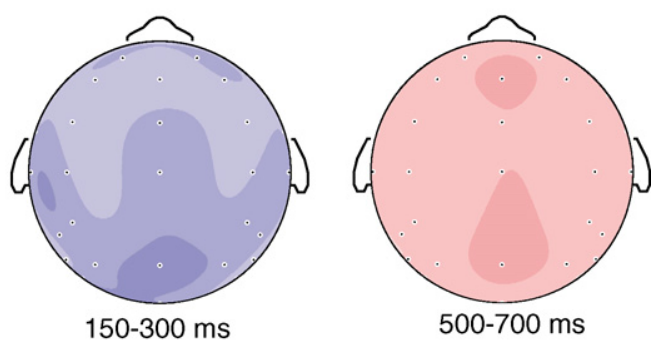

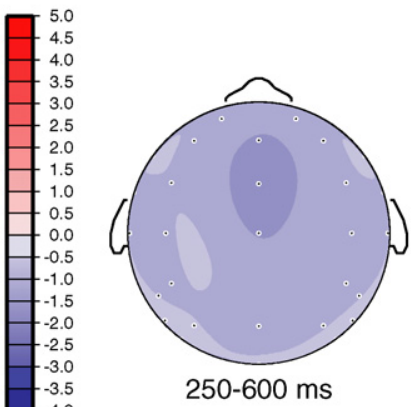

$250-600 \mathrm{~ms}$

Fig. 5. Grand average waveforms for two representative electrodes (Fz, Pz) and isopotential voltage maps, time-locked to the acoustic onset of the verb (A) and sentence-final noun (B) for the normal control subjects $(N=15)$ for the semantically reversible active sentences. Dotted line is used for the mismatching sentences, solid line for the matching counterparts. Isopotential voltage maps were based on mean amplitude differences in the specified latency windows. Positive polarity effects are in red, negative-going effects are in blue. Scale values are in $\mu \mathrm{V}$. (For interpretation of the references to colour in this figure legend, the reader is referred to the web version of the article.)

between a picture and a sentence as early as possible. Table 5 shows the relevant statistical results for the different time epochs, belonging to this condition.

The critical auxiliary verb in the mismatching condition (see Fig. 6A) elicited mainly at centro-posterior sites a negativegoing wave in the $200-400 \mathrm{~ms}$ epoch. The negativity was significant in an ANOVA that included only posterior electrodes (see Table 5). The negative effect was followed by a positive shift starting at about $400 \mathrm{~ms}$ and extending up to $900 \mathrm{~ms}$. For anterior sites at which the negative-going wave was absent, this positive shift started earlier and was present in, approximately, the $300-750 \mathrm{~ms}$ latency range. The positivity was significant in an omnibus ANOVA in the $450-700 \mathrm{~ms}$ latency window (see Table 5).

The sentence-final noun (see Fig. 6B) elicited mainly for frontal electrodes a small but sustained negative shift, starting at around $400 \mathrm{~ms}$. However, this effect was not significant (see Table 5).

\subsubsection{Summary of ERP data normal control subjects}

The ERP data of the normal elderly controls showed on-line sensitivity to a mismatch between a picture and a sentence. As soon as the (auxiliary) verb was heard, the ERP signature showed a negative effect. This effect was followed by a positive shift, 
(A) Semantically reversible (passive)

$\mathrm{Fz}$

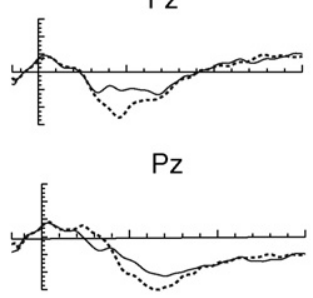

Voltage [uV]
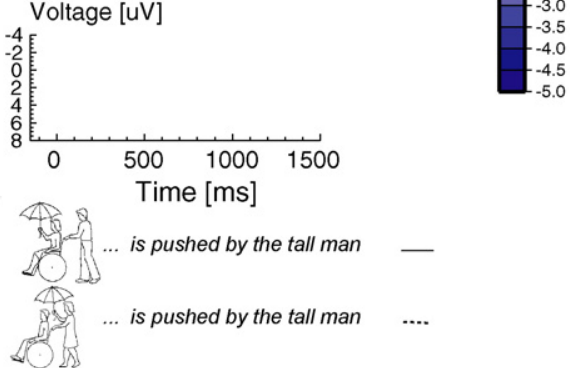

(B)

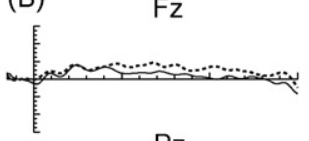

$\mathrm{Pz}$
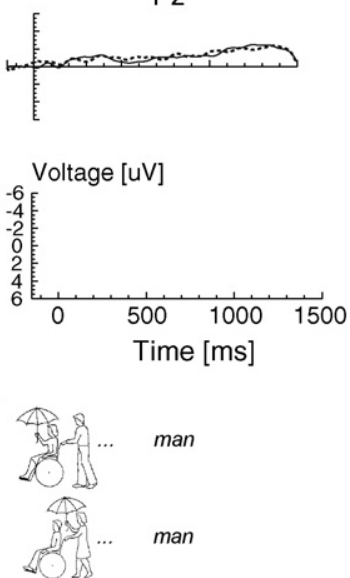

Normal Controls $(N=15)$

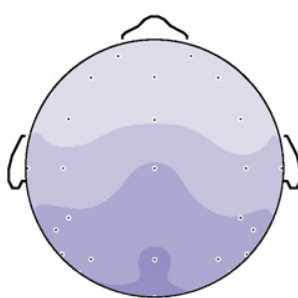

250-350 ms

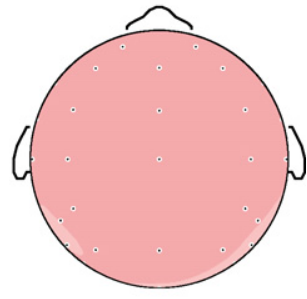

$450-700 \mathrm{~ms}$

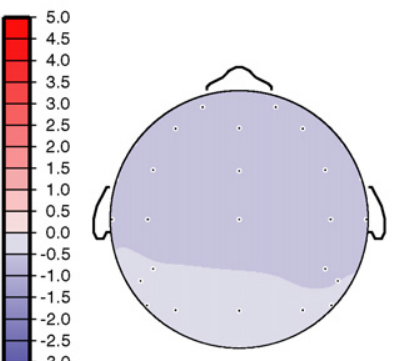

400-700 ms

Fig. 6. Grand average waveforms for two representative electrodes $(\mathrm{Fz}, \mathrm{Pz})$ and isopotential voltage maps, time-locked to the acoustic onset of the auxiliary verb (A) and sentence-final noun (B) for the normal control subjects $(N=15)$ for the semantically reversible passive sentences. Dotted line is used for the mismatching sentences, solid line for the matching counterparts. Isopotential voltage maps were based on mean amplitude differences in the specified latency windows. Positive polarity effects are in red, negative-going effects are in blue. Scale values are in $\mu \mathrm{V}$. (For interpretation of the references to colour in this figure legend, the reader is referred to the web version of the article.)

Table 5

Semantically reversible passive sentences for normal control subjects: mean ERP amplitude ANOVAs in the specified latency ranges

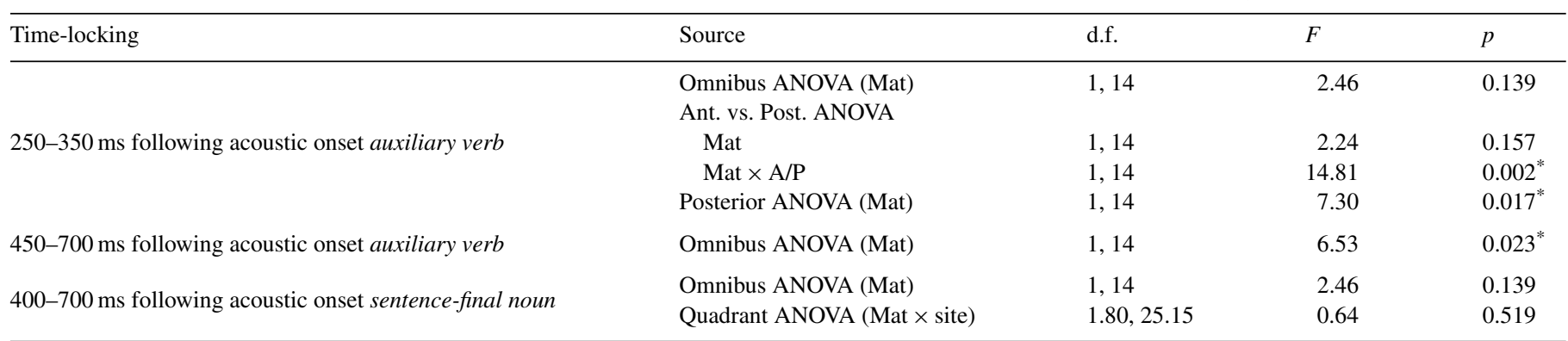

Note. Mat: matching type (matching vs. mismatching). ${ }^{*} p \leq 0.05$. Omnibus ANOVA: 29 electrodes; quadrant ANOVA: $4 \times 5$ electrodes; anterior vs. posterior ANOVA: $2 \times 10$ electrodes; posterior ANOVA: 10 electrodes. Degrees of freedom (d.f.) are Huynh-Feldt corrected. 
except for the semantically reversible active sentences. In addition, for the active sentences, a negative effect was also elicited by the sentence-final noun.

\subsection{RH patients}

\subsubsection{Semantically irreversible active sentences}

Fig. 7 displays for the semantically irreversible active sentences grand average waveforms for two representative electrodes and isopotential voltage maps, time-locked to the acoustic onset of the critical verb (upper panel) and sentence-final noun (lower panel). Table 6 shows the relevant statistical results for the different time epochs, belonging to this condition.

A positive effect is elicited by the critical verb in the mismatching condition between approximately 450 and $1000 \mathrm{~ms}$ (see Fig. 7A). This positive-going waveform does not seem to be preceded by a clear negative effect. Statistical analysis corroborates this latter observation: in the 100-200 ms latency range there is no overall significant effect of matching (see Table 6). The positive shift, tested in an omnibus ANOVA in the 500-800 ms latency window, failed to reach significance level. However, an ANOVA in which only the posterior sites were included showed a marginally significant effect of matching $(p<0.055$; see Table 6).

The sentence-final noun (see Fig. 7B) elicited a broad and sustained negative shift in the mismatching condition, starting at $200 \mathrm{~ms}$ post-onset and continuing throughout the remaining epoch. This effect was significant (see Table 6).

\subsubsection{Semantically reversible active sentences}

Fig. 8 displays for the semantically irreversible active sentences grand average waveforms for two representative electrodes and isopotential voltage maps, time-locked to the acoustic onset of the critical verb (upper panel) and sentence-final noun
(A) Semantically irreversible (active)
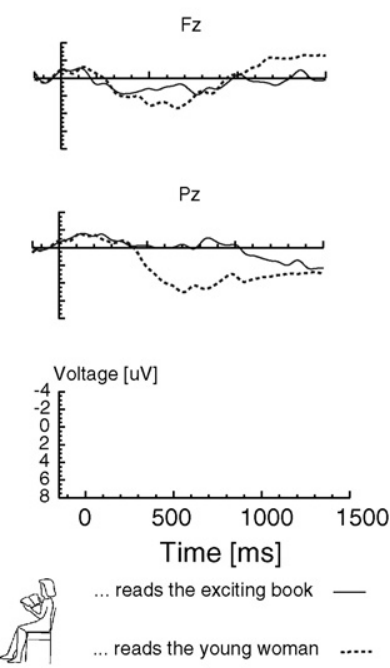

(B)

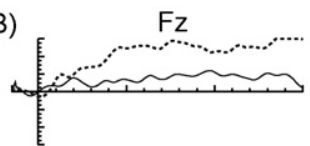

$\mathrm{Pz}$
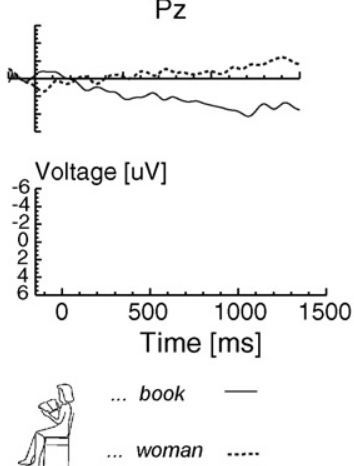

RH patients $(N=8)$

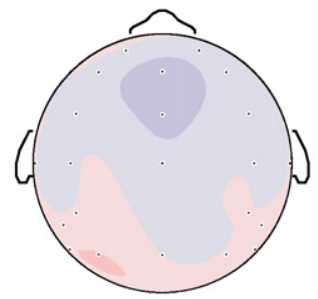

100-200 ms

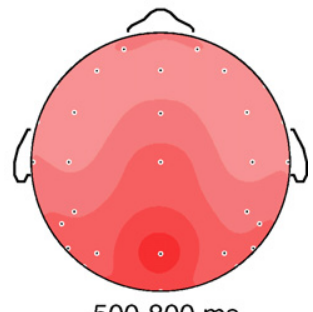

$500-800 \mathrm{~ms}$

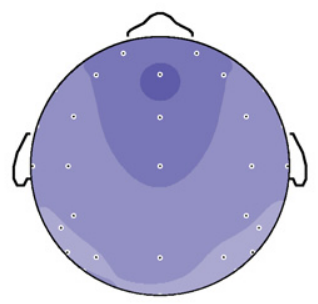

$250-600 \mathrm{~ms}$

Fig. 7. Grand average waveforms for two representative electrodes (Fz, Pz) and isopotential voltage maps, time-locked to the acoustic onset of the verb (A) and sentence-final noun $(\mathrm{B})$ for the RH patients $(N=8)$ for the semantically irreversible active sentences. Dotted line is used for the mismatching sentences, solid line for the matching counterparts. Isopotential voltage maps were based on mean amplitude differences in the specified latency windows. Positive polarity effects are in red, negative-going effects are in blue. Scale values are in $\mu \mathrm{V}$. (For interpretation of the references to colour in this figure legend, the reader is referred to the web version of the article.) 
Table 6

Semantically irreversible active sentences for RH patients: mean ERP amplitude ANOVAs in the specified latency ranges

\begin{tabular}{|c|c|c|c|c|}
\hline Time-locking & Source & d.f. & $F$ & $p$ \\
\hline $100-200 \mathrm{~ms}$ following acoustic onset verb & $\begin{array}{l}\text { Omnibus ANOVA (Mat) } \\
\text { Quadrant ANOVA (Mat } \times \text { site) }\end{array}$ & $\begin{array}{l}1,7 \\
3,21\end{array}$ & $\begin{array}{l}0.15 \\
1.55\end{array}$ & $\begin{array}{l}0.708 \\
0.231\end{array}$ \\
\hline
\end{tabular}

Note. Mat: matching type (matching vs. mismatching). ${ }^{*} p<0.05$ Omnibus ANOVA: 29 electrodes; quadrant ANOVA: $4 \times 5$ electrodes; anterior vs. posterior ANOVA: $2 \times 10$ electrodes; posterior ANOVA: 10 electrodes. Degrees of freedom (d.f.) are Huynh-Feldt corrected.

(A) Semantically reversible (active)

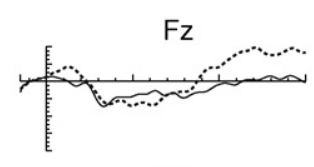

$\mathrm{PZ}$
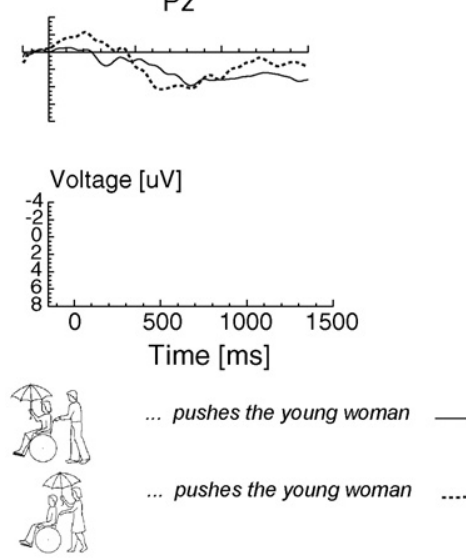

(B)
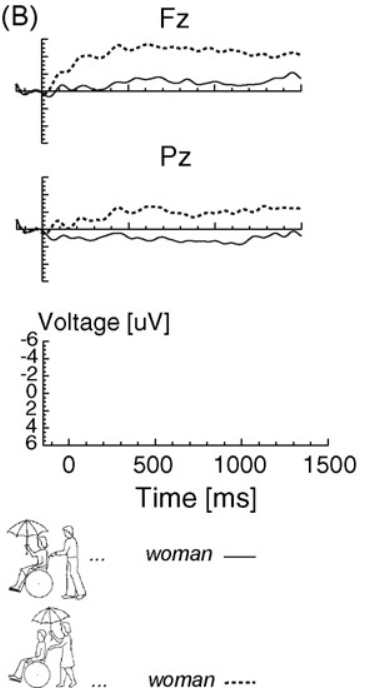

RH patients $(N=8)$

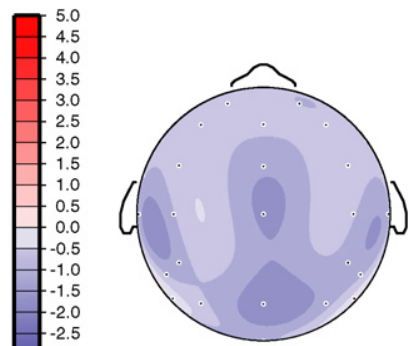

$150-300 \mathrm{~ms}$

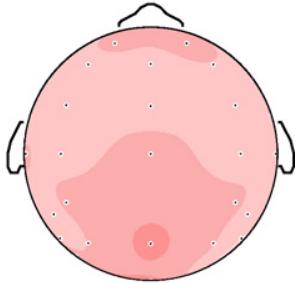

$500-700 \mathrm{~ms}$

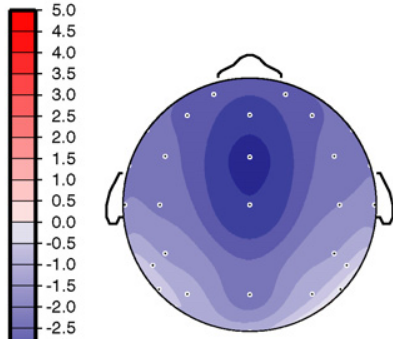

$250-600 \mathrm{~ms}$

Fig. 8. Grand average waveforms for two representative electrodes $(\mathrm{Fz}, \mathrm{Pz})$ and isopotential voltage maps, time-locked to the acoustic onset of the verb (A) and sentence-final noun (B) for the RH patients $(N=8)$ for the semantically reversible active sentences. Dotted line is used for the mismatching sentences, solid line for the matching counterparts. Isopotential voltage maps were based on mean amplitude differences in the specified latency windows. Positive polarity effects are in red, negative-going effects are in blue. Scale values are in $\mu \mathrm{V}$. (For interpretation of the references to colour in this figure legend, the reader is referred to the web version of the article.) 
Table 7

Semantically reversible active sentences for RH patients: mean ERP amplitude ANOVAs in the specified latency ranges

\begin{tabular}{|c|c|c|c|c|}
\hline Time-locking & Source & d.f. & $F$ & $p$ \\
\hline \multirow{2}{*}{$150-300 \mathrm{~ms}$ following acoustic onset verb } & Omnibus ANOVA (Mat) & 1,7 & 4.77 & 0.065 \\
\hline & Quadrant ANOVA (Mat × site) & $1.64,11.46$ & 2.11 & 0.169 \\
\hline \multirow{4}{*}{$500-700 \mathrm{~ms}$ following acoustic onset verb } & Omnibus ANOVA (Mat) & 1,7 & 1.31 & 0.289 \\
\hline & Quadrant ANOVA (Mat × site) & $2.05,14.33$ & 6.56 & $0.009^{*}$ \\
\hline & Ant. vs. Post. ANOVA $($ Mat $\times$ A/P) & 1,7 & 13.27 & $0.008^{*}$ \\
\hline & Posterior ANOVA (Mat) & 1,7 & 3.00 & 0.127 \\
\hline \multirow{3}{*}{$250-600 \mathrm{~ms}$ following acoustic onset sentence-final noun } & Omnibus ANOVA (Mat) & 1,7 & 4.25 & 0.078 \\
\hline & Ant. vs. Post. ANOVA (Mat $\times$ A/P) & 1,7 & 6.54 & $0.038^{*}$ \\
\hline & Anterior ANOVA (Mat) & 1,7 & 6.76 & $0.035^{*}$ \\
\hline
\end{tabular}

Note. Mat: matching type (matching vs. mismatching). ${ }^{*} p \leq 0.05$. Omnibus ANOVA: 29 electrodes; quadrant ANOVA: $4 \times 5$ electrodes; anterior vs. posterior ANOVA: $2 \times 10$ electrodes; posterior ANOVA: 10 electrodes; anterior ANOVA: 10 electrodes. Degrees of freedom (d.f.) are Huynh-Feldt corrected.

(lower panel). Table 7 shows the relevant statistical results for the different time epochs, belonging to this condition.

The critical verb in the mismatching condition elicited a negative-going wave that was present approximately between 50 and $300 \mathrm{~ms}$ for anterior sites (see Fig. 8A). For posterior sites the negative effect lasted somewhat longer (until $450 \mathrm{~ms}$ ). This negativity was marginally significant (see Table 7). The negative-going wave was followed by a positive shift starting at about $350 \mathrm{~ms}$ for anterior and at about $450 \mathrm{~ms}$ for posterior sites, and extending up to $900 \mathrm{~ms}$ for posterior sites. The positive shift was largest over posterior sites. However, an analysis in which only posterior electrodes were included failed to reach significance level (see Table 7).

The sentence-final noun in the mismatching condition elicited a broad, widely distributed sustained negative shift (see Fig. 8B), which was largest over anterior sites. An ANOVA that included only the anterior electrode sites revealed a significant matching effect (see Table 7).

\subsubsection{Semantically reversible passive sentences}

Fig. 9 displays grand average waveforms for two representative electrodes and isopotential voltage maps, time-locked to the acoustic onset of the auxiliary verb (upper panel) and sentencefinal noun (lower panel). Table 8 shows the relevant statistical results for the different time epochs, belonging to this condition.

The critical auxiliary verb in the mismatching condition (see Fig. 9A) elicited mainly for posterior electrode sites a negativegoing wave in, approximately, the $200-400 \mathrm{~ms}$ epoch followed by a positive shift starting at about $400 \mathrm{~ms}$ and extending up to $1300 \mathrm{~ms}$. The negative shift was not significant (see Table 8). The positive shift was marginally significant in an omnibus ANOVA for the $600-850 \mathrm{~ms}$ window (see Table 8 ).

The sentence-final noun (see Fig. 9B) elicited in the mismatching condition a broad and sustained negative shift, clearly manifest at $100 \mathrm{~ms}$ post-onset and continuing throughout the remaining epoch. This effect was significant (see Table 8).

\subsubsection{Between-subjects analyses (RH patients versus normal controls)}

Results of the RH patients were statistically compared to the normal controls in analyses with group of subjects as additional factor (see Table 9). For both the semantically irreversible active and the reversible active sentences no significant interactions between group of subjects and matching were obtained. Thus, the overall pattern of results for these conditions was statistically not different between the normal controls and the RH patients. This was also the case for the semantically reversible passive sentences except for the sentence-final noun, due to the presence of the large sustained negative shift in the RH patients while this was not the case for the normal control subjects. In sum, the ERP results of the RH patients bear clear resemblance to the data of the normal controls.

\subsection{Broca patients}

\subsubsection{Semantically irreversible active sentences}

Fig. 10 displays for the semantically irreversible active sentences grand average waveforms for two representative electrodes and isopotential voltage maps, time-locked to the acoustic onset of the critical verb (upper panel) and sentence-final noun (lower panel). Table 10 shows the relevant statistical results for the different time epochs, belonging to this condition.

The negative effect in the early time window was not significant (see Fig. 10A and Table 10). A positive-going waveform with a posterior distribution appears to be present in these Broca patients, but the onset of the effect (at around $700 \mathrm{~ms}$ ) is considerably delayed with respect to the control groups. The omnibus ANOVA in the $500-800 \mathrm{~ms}$ latency window did not reveal a significant effect of matching (see Table 10). However, when the effect was tested in a later window (800-1100 ms), a significant effect was found for posterior electrode sites (see Table 10).

For the sentence-final noun (see Fig. 10B), mainly at posterior sites, a positive shift in the $0-500 \mathrm{~ms}$ latency range is visible, which possibly reflects the delayed ERP response to the critical verb. It is only from $600 \mathrm{~ms}$ onwards that the waveforms show a negative shift for the mismatching condition. However, none of these effects were significant (see Table 10).

\subsubsection{Semantically reversible active sentences}

Fig. 11 displays for the semantically irreversible active sentences grand average waveforms for two representative electrodes and isopotential voltage maps, time-locked to the acoustic 
(A) Semantically reversible (passive)
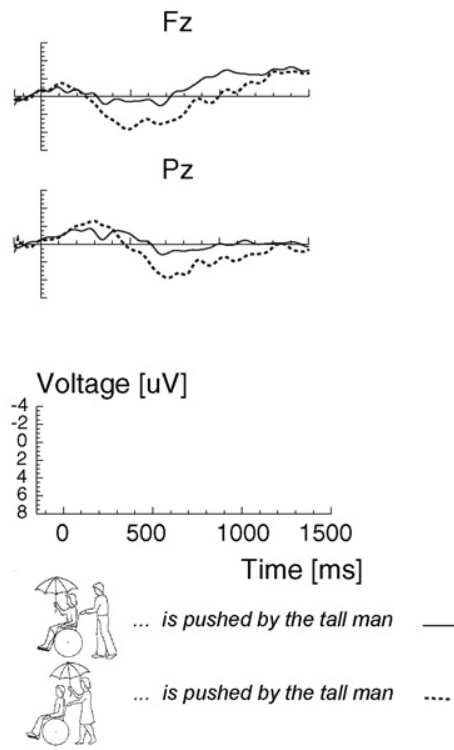

(B)
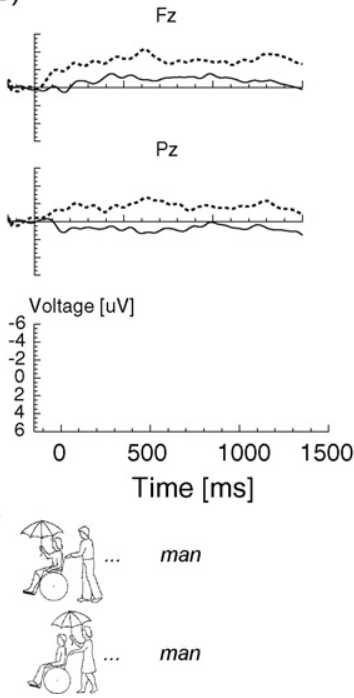

RH patients $(N=8)$

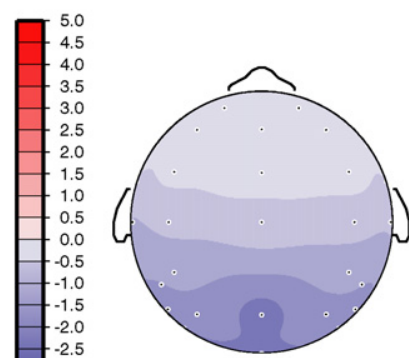

250-350 ms

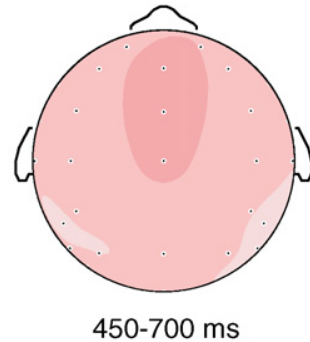

$450-700 \mathrm{~ms}$

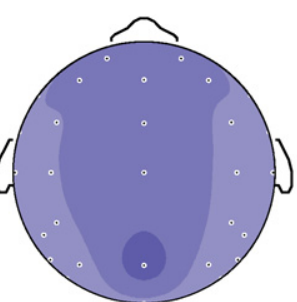

400-700 ms

Fig. 9. Grand average waveforms for two representative electrodes (Fz, Pz) and isopotential voltage maps, time-locked to the acoustic onset of the auxiliary verb (A) and sentence-final noun (B) for the RH patients $(N=8)$ for the semantically reversible passive sentences. Dotted line is used for the mismatching sentences, solid line for the matching counterparts. Isopotential voltage maps were based on mean amplitude differences in the specified latency windows. Positive polarity effects are in red, negative-going effects are in blue. Scale values are in $\mu \mathrm{V}$. (For interpretation of the references to colour in this figure legend, the reader is referred to the web version of the article.)

Table 8

Semantically reversible passive sentences for RH patients: mean ERP amplitude ANOVAs in the specified latency ranges

\begin{tabular}{llrrr}
\hline Time-locking & Source & d.f. & $F$ & $p$ \\
\hline 250-350 ms following acoustic onset auxiliary verb & Omnibus ANOVA (Mat) & 1,7 & 0.02 & 0.884 \\
450-700 ms following acoustic onset auxiliary verb & Omnibus ANOVA (Mat) & 1,7 & 3.39 & 0.108 \\
& Omnibus ANOVA (Mat) & 1,7 & 0.95 \\
600-850 ms following acoustic onset auxiliary verb & Left electrodes (Mat) & 1,7 & 5.82 & 0.062 \\
& Omnibus ANOVA (Mat) & 1,7 & 22.98 & $0.002^{*}$ \\
400-700 ms following acoustic onset sentence-final noun & Quadrant ANOVA (Mat $\times$ site) & 3,21 & 0.20 \\
\hline
\end{tabular}

Note. Mat: matching type (matching vs. mismatching). ${ }^{*} p \leq 0.05$. Omnibus ANOVA: 29 electrodes; quadrant ANOVA: $4 \times 5$ electrodes; anterior vs. posterior ANOVA: $2 \times 10$ electrodes; posterior ANOVA: 10 electrodes. 
Table 9

Between subjects ANOVAs in specified latency ranges: normal controls vs. RH patients

\begin{tabular}{|c|c|c|c|c|}
\hline Time-locking & Source & d.f. & $F$ & $p$ \\
\hline \multicolumn{5}{|l|}{ Sem. irreversible actives } \\
\hline \multirow{4}{*}{$100-200 \mathrm{~ms}$ following verb } & Omnibus ANOVA & & & \\
\hline & Group & 1,21 & 0.00 & 0.979 \\
\hline & Mat & 1,21 & 3.82 & 0.064 \\
\hline & Group $\times$ Mat & 1,21 & 1.78 & 0.197 \\
\hline \multirow{4}{*}{$500-800 \mathrm{~ms}$ following verb } & Omnibus ANOVA & & & \\
\hline & Group & 1,21 & 3.04 & 0.096 \\
\hline & Mat & 1,21 & 10.91 & $0.003^{*}$ \\
\hline & Group $\times$ Mat & 1,21 & 0.10 & 0.749 \\
\hline \multirow{4}{*}{ 250-600 ms following sentence-final noun } & Omnibus ANOVA & & & \\
\hline & Group & 1,21 & 0.09 & 0.772 \\
\hline & Mat & 1,21 & 16.03 & $0.001^{* *}$ \\
\hline & Group $\times$ Mat & 1,21 & 0.47 & 0.502 \\
\hline \multicolumn{5}{|l|}{ Sem. reversible actives } \\
\hline \multirow{4}{*}{$150-300 \mathrm{~ms}$ following verb } & Omnibus ANOVA & & & \\
\hline & Group & 1,21 & 1.00 & 0.330 \\
\hline & Mat & 1,21 & 10.78 & $0.004^{*}$ \\
\hline & Group $\times$ Mat & 1,21 & 0.01 & 0.915 \\
\hline \multirow{4}{*}{ 500-700 ms following verb } & Omnibus ANOVA & & & \\
\hline & Group & 1,21 & 2.33 & 0.142 \\
\hline & Mat & 1,21 & 3.56 & 0.073 \\
\hline & Group $\times$ Mat & 1,21 & 0.12 & 0.735 \\
\hline \multirow{4}{*}{ 250-600 ms following sentence-final noun } & Omnibus ANOVA & & & \\
\hline & Group & 1,21 & 4.69 & $0.042^{*}$ \\
\hline & Mat & 1,21 & 7.57 & $0.012^{*}$ \\
\hline & Group $\times$ Mat & 1,21 & 0.22 & 0.645 \\
\hline \multicolumn{5}{|l|}{ Sem. reversible passives } \\
\hline \multirow{8}{*}{ 250-350 ms following auxiliary verb } & Omnibus ANOVA & & & \\
\hline & Group & 1,21 & 8.47 & $0.008^{*}$ \\
\hline & Mat & 1,21 & 0.90 & 0.353 \\
\hline & Group $\times$ Mat & 1,21 & 0.42 & 0.523 \\
\hline & Posterior ANOVA & & & \\
\hline & Group & 1,21 & 8.56 & $0.008^{*}$ \\
\hline & Mat & 1,21 & 5.48 & $0.029^{*}$ \\
\hline & Group $\times$ Mat & 1,21 & 0.26 & 0.618 \\
\hline \multirow{4}{*}{$450-700 \mathrm{~ms}$ following auxiliary verb } & Omnibus ANOVA & & & \\
\hline & Group & 1,21 & 6.43 & $0.019^{*}$ \\
\hline & Mat & 1,21 & 9.85 & $0.005^{*}$ \\
\hline & Group $\times$ Mat & 1,21 & 0.34 & 0.564 \\
\hline \multirow{4}{*}{ 400-700 ms following sentence-final noun } & Omnibus ANOVA & & & \\
\hline & Group & 1,21 & 0.03 & 0.864 \\
\hline & Mat & 1,21 & 20.06 & $0.000^{* *}$ \\
\hline & Group $\times$ Mat & 1,21 & 6.49 & $0.019^{*}$ \\
\hline
\end{tabular}

Note. Mat: matching type (matching vs. mismatching). ${ }^{*} p \leq 0.05 ;{ }^{* *} p \leq 0.001$. Omnibus ANOVA: 29 electrodes; posterior ANOVA: 10 electrodes.

onset of the critical verb (upper panel) and sentence-final noun (lower panel). Table 11 shows the relevant statistical results for the different time epochs, belonging to this condition.

The critical verb in the mismatching condition (see Fig. 11A) elicited no significant matching effects (see Table 11).

For the sentence-final noun (see Fig. 11B) a significant interaction between matching and posterior left/posterior right regions was present. The negative shift was, in an ANOVA that included only right posterior electrode sites, significant (see Table 11).

\subsubsection{Semantically reversible passive sentences}

Fig. 12 displays grand average waveforms for two representative electrodes and isopotential voltage maps, time-locked to the acoustic onset of the auxiliary verb (upper panel) and sentence-final noun (lower panel). Table 12 shows the relevant statistical results for the different time epochs, belonging to this condition.

As can be seen in Fig. 12, the Broca's aphasics did not show reliable ERP effects for this sentence type. Both the critical auxiliary verb and the sentence-final noun in the mismatching 
(A) Semantically irreversible (active)
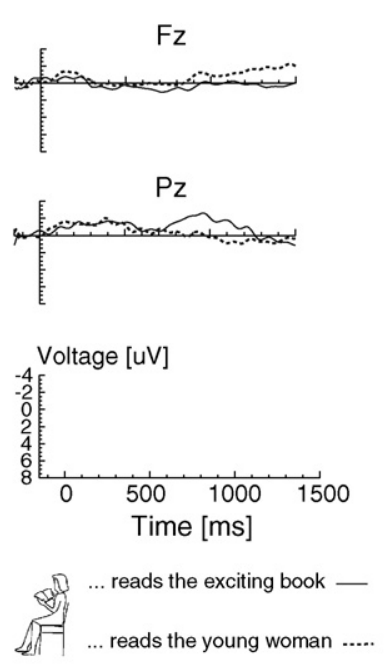

(B)

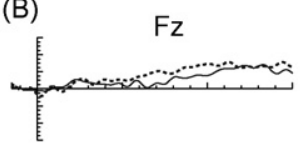

$\mathrm{Pz}$
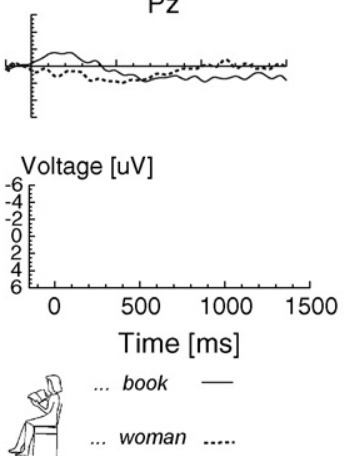

Broca patients $(N=10)$

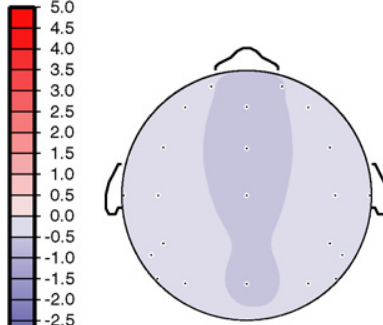

$100-200 \mathrm{~ms}$

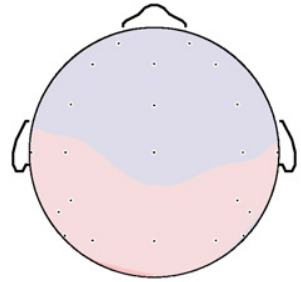

$500-800 \mathrm{~ms}$

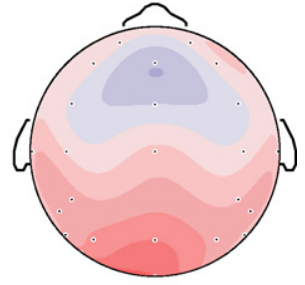

800-1100 ms

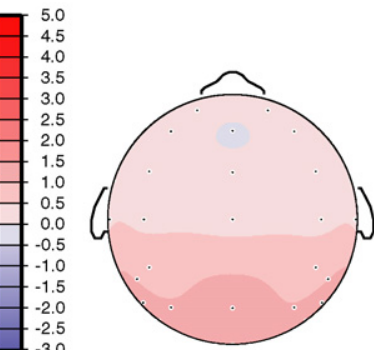

$250-600 \mathrm{~ms}$

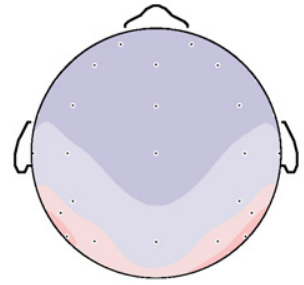

650-1000 ms

Fig. 10. Grand average waveforms for two representative electrodes (Fz, Pz) and isopotential voltage maps, time-locked to the acoustic onset of the verb (A) and sentence-final noun (B) for the Broca patients $(N=10)$ for the semantically irreversible active sentences. Dotted line is used for the mismatching sentences, solid line for the matching counterparts. Isopotential voltage maps were based on mean amplitude differences in the specified latency windows. Positive polarity effects are in red, negative-going effects are in blue. Scale values are in $\mu \mathrm{V}$. (For interpretation of the references to colour in this figure legend, the reader is referred to the web version of the article.)

Table 10

Semantically irreversible active sentences for Broca patients: mean ERP amplitude ANOVAs in the specified latency ranges

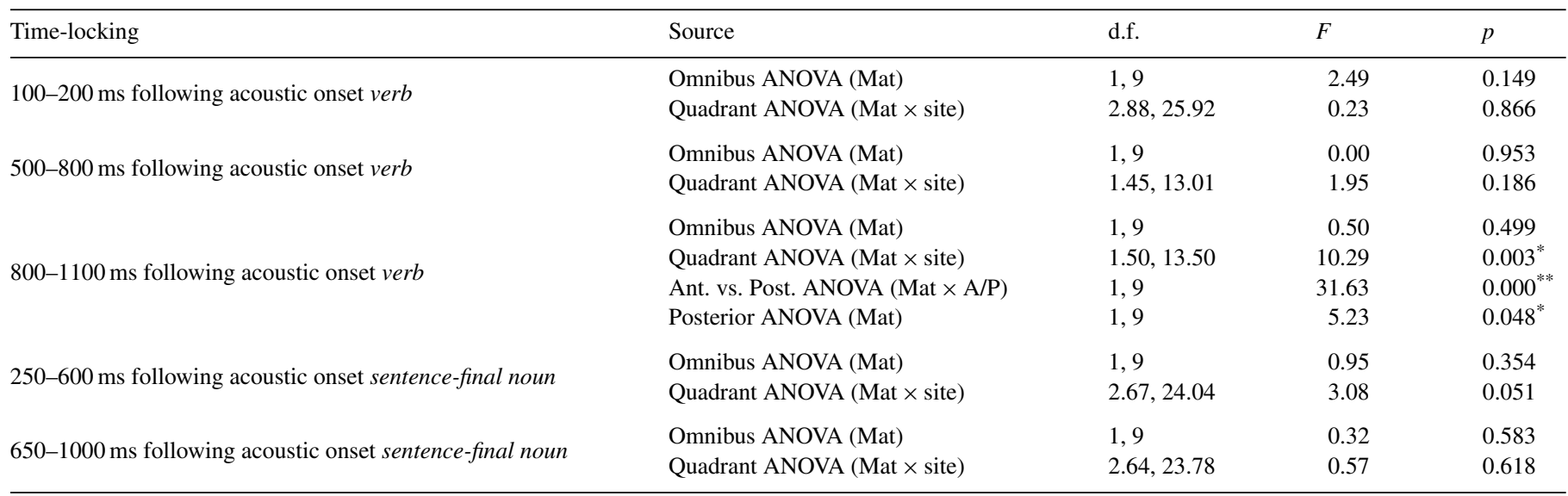

Note. Mat: matching type (matching vs. mismatching). ${ }^{*} p \leq 0.05 ;{ }^{* *} p \leq 0.001$. Omnibus ANOVA: 29 electrodes; quadrant ANOVA: $4 \times 5$ electrodes; anterior vs. posterior ANOVA: $2 \times 10$ electrodes; posterior ANOVA: 10 electrodes. Degrees of freedom (d.f.) are Huynh-Feldt corrected. 
(A) Semantically reversible (active)

$\mathrm{Fz}$

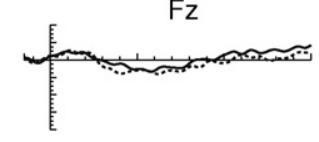

$\mathrm{PZ}$
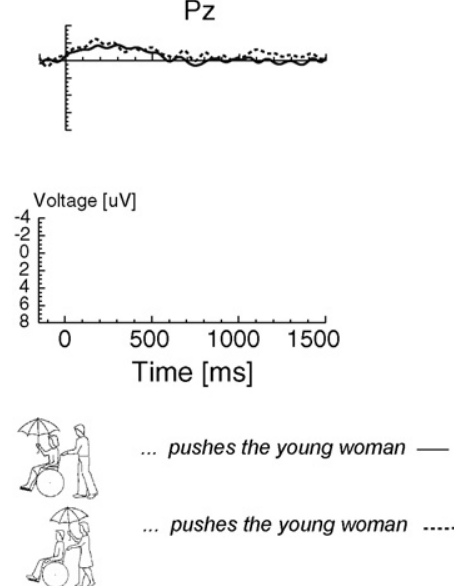

(B)

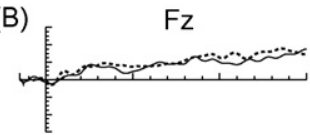

$\mathrm{Pz}$
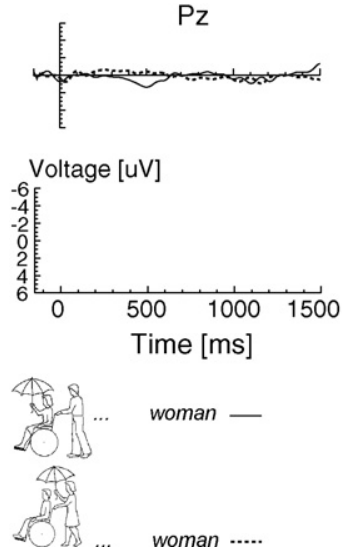

Broca patients $(N=10)$

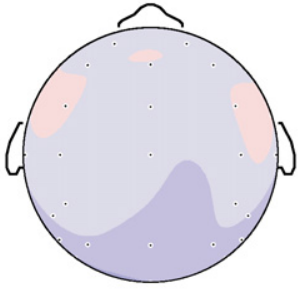

150-300 ms

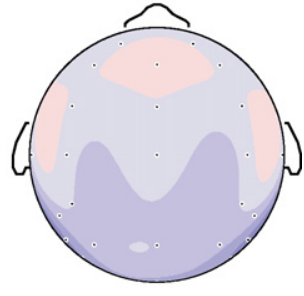

$500-700 \mathrm{~ms}$

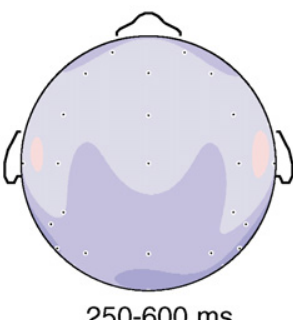

250-600 ms

Fig. 11. Grand average waveforms for two representative electrodes ( Fz, Pz) and isopotential voltage maps, time-locked to the acoustic onset of the verb (A) and sentence-final noun $(\mathrm{B})$ for the Broca patients $(N=10)$ for the semantically reversible active sentences. Dotted line is used for the mismatching sentences, solid line for the matching counterparts. Isopotential voltage maps were based on mean amplitude differences in the specified latency windows. Positive polarity effects are in red, negative-going effects are in blue. Scale values are in $\mu \mathrm{V}$. (For interpretation of the references to colour in this figure legend, the reader is referred to the web version of the article.)

condition elicited in the Broca patients no significant effects (see Table 12).

\subsubsection{Between-subjects analyses (Broca patients versus normal controls)}

Results of the Broca patients were statistically compared to the normal controls in analyses with group of subjects as additional factor (see Table 13).

For the semantically irreversible active sentences the group of subjects by matching interaction was significant for the $500-800 \mathrm{~ms}$ epoch. This interaction was due to the delay of the positive effect in the Broca patients relative to the normal controls. Also the omnibus ANOVA for the sentence-final latency window resulted in a significant group of subjects by match- ing interaction. Thus, the pattern of ERP results of the Broca patients for the semantically irreversible active sentences was clearly different from the normal controls.

For the semantically reversible active sentences, highly significant group of subjects effects were obtained. The interaction between group of subjects and matching approached significance for posterior sites in the 500-700 ms window.

For the semantically reversible passive sentences, there was a (marginally) significant group of subjects by matching interaction for the $250-350 \mathrm{~ms}$ latency window following the auxiliary verb over posterior electrodes.

In sum, the ERP results of the Broca patients were clearly different from the normal controls. Whereas for the normal controls ERP effects were obtained at the position of the verb for 
Table 11

Semantically reversible active sentences for Broca patients: mean ERP amplitude ANOVAs in the specified latency ranges

\begin{tabular}{|c|c|c|c|c|}
\hline Time-locking & Source & d.f. & $F$ & $p$ \\
\hline \multirow{4}{*}{$150-300 \mathrm{~ms}$ following acoustic onset verb } & Omnibus ANOVA (Mat) & 1,9 & 0.84 & 0.383 \\
\hline & Quadrant ANOVA (Mat $\times$ site) & $1.97,17.74$ & 3.58 & $0.050^{*}$ \\
\hline & Ant. vs. Post. ANOVA (Mat $\times$ site) & 1,9 & 4.17 & 0.071 \\
\hline & Posterior ANOVA (Mat) & 1,9 & 2.57 & 0.143 \\
\hline \multirow{4}{*}{$500-700 \mathrm{~ms}$ following acoustic onset verb } & Omnibus ANOVA (Mat) & 1,9 & 0.39 & 0.547 \\
\hline & Quadrant (Mat $\times$ site $)$ & 3,27 & 8.79 & $0.000^{* *}$ \\
\hline & Ant. vs. Post. ANOVA (Mat $\times$ A/P) & 1,9 & 9.39 & $0.013^{*}$ \\
\hline & Posterior ANOVA (Mat) & 1,9 & 2.02 & 0.189 \\
\hline \multirow{4}{*}{$250-600 \mathrm{~ms}$ following acoustic onset sentence-final noun } & Omnibus ANOVA (Mat) & 1,9 & 0.79 & 0.396 \\
\hline & Quadrant ANOVA (Mat $\times$ site $)$ & 3,27 & 4.10 & $0.016^{*}$ \\
\hline & Post. L. vs. Post. R. ANOVA (Mat $\times$ site) & 1,9 & 8.67 & $0.016^{*}$ \\
\hline & Posterior right ANOVA (Mat) & 1,9 & 8.69 & $0.016^{*}$ \\
\hline
\end{tabular}

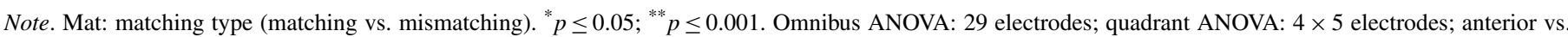

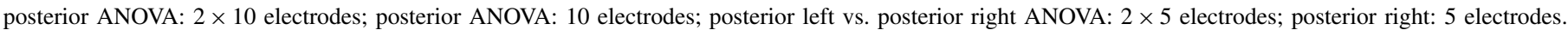
Degrees of freedom (d.f.) are Huynh-Feldt corrected.

Table 12

Semantically reversible passive sentences for Broca patients: mean ERP amplitude ANOVAs in the specified latency ranges

\begin{tabular}{|c|c|c|c|c|}
\hline Time-locking & Source & d.f. & $F$ & $p$ \\
\hline $250-350 \mathrm{~ms}$ following acoustic onset auxiliary verb & Omnibus ANOVA (Mat) & 1,9 & 0.01 & 0.932 \\
\hline 450-700 ms following acoustic onset auxiliary verb & Omnibus ANOVA (Mat) & 1,9 & 1.33 & 0.278 \\
\hline $400-700 \mathrm{~ms}$ following acoustic onset sentence-final noun & Omnibus ANOVA (Mat) & 1,9 & 0.70 & 0.426 \\
\hline 200-500 ms following acoustic onset sentence-final noun & Omnibus ANOVA (Mat) & 1,9 & 0.76 & 0.405 \\
\hline
\end{tabular}

Note. Mat: matching type (matching vs. mismatching). Omnibus ANOVA: 29 electrodes.

all sentence types, the Broca patients showed a reduced and delayed ERP effect time-locked to the onset of the verb only for the semantically irreversible sentences.

\section{Discussion}

The present study was designed to investigate possible impairments in the on-line thematic role assignment in patients with Broca's aphasia. For that purpose, ERPs were recorded while subjects were engaged in sentence-picture matching. In addition, reaction time and accuracy were measured. In Table 14, the ERP results of the experiment are summarized for the three different subject groups.

The ERP data of the normal elderly controls showed on-line sensitivity to a mismatch between a picture and a sentence. As soon as the (auxiliary) verb was heard, the ERP signature showed a negative effect. Except for the semantically reversible active sentences, this effect was followed by a positive shift. In addition, for the active sentences, a negative effect was also elicited by the sentence-final noun. The size and to a certain extent also the topographic distributions of the effects differed as function of sentence type. Before focussing on the immediate character of the thematic role assignment process in the normal controls, we will first address the issue of what kind of ERP componentry seems to be involved in these effects.

It is not immediately clear how to label the early negative effect that was found in the three sentence conditions, also because of the fact that the topographic distribution of the early negative effects differed along sentence type. The anterior distribution of the effect for the semantically irreversible sentences (sentence type I) makes it at first sight less likely that this effect reflects an N400 effect, which has characteristically a centroposterior distribution. Furthermore, the onset of the effect for sentence type I seems also rather early for being an N400. However, the early negative effects found for both types of reversible sentences (types II and III) do have a posterior maximum. We cannot exclude the possibility that the underlying distribution of the early negative effect for sentence type I also has a posterior maximum, but that the partly overlapping posteriorly distributed positivity has masked the posterior contribution of the negative shift.

Interestingly, also D'Arcy and Connolly (1999) found, with an ERP version of the token test, an early negative effect (followed by a positive shift) when a spoken sentence contained a word that incorrectly described the previous studied visuospatial animation (for instance "touched the small blue circle and the large green square" instead of "touched the small blue circle and the large red square"). D'Arcy and Connolly interpreted this early negative effect as an $\mathrm{N} 2 \mathrm{~b}$ reflecting a deviation in the incoming speech stimuli from an active cognitive template formed from the visual information. In this view, the early negative effect reflected primarily attentional detection rather than language processing. Certainly, our present experiment shares task requirements with the experiment of D'Arcy and Connolly, namely analysis of visual information, maintenance of that information in working memory, and matching of incoming spoken 
Table 13

Between subjects ANOVAs in specified latency ranges: normal controls vs. Broca patients

\begin{tabular}{|c|c|c|c|c|}
\hline Time-locking & Source & d.f. & $F$ & $p$ \\
\hline \multicolumn{5}{|l|}{ Sem. irreversible actives } \\
\hline \multirow{4}{*}{$100-200 \mathrm{~ms}$ following verb } & Omnibus ANOVA & & & \\
\hline & Group & 1,23 & 0.00 & 0.979 \\
\hline & Mat & 1,23 & 8.82 & $0.007^{*}$ \\
\hline & Group $\times$ Mat & 1,23 & 0.31 & 0.584 \\
\hline \multirow{4}{*}{$500-800 \mathrm{~ms}$ following verb } & Omnibus ANOVA & & & \\
\hline & Group & 1,23 & 15.27 & $0.001^{* *}$ \\
\hline & Mat & 1,23 & 4.05 & 0.056 \\
\hline & Group $\times$ Mat & 1,23 & 4.28 & $0.050^{*}$ \\
\hline \multirow{4}{*}{$250-600 \mathrm{~ms}$ following sentence-final noun } & Omnibus ANOVA & & & \\
\hline & Group & 1,23 & 3.54 & 0.073 \\
\hline & Mat & 1,23 & 1.59 & 0.219 \\
\hline & Group $\times$ Mat & 1,23 & 6.93 & $0.015^{*}$ \\
\hline \multicolumn{5}{|l|}{ Sem. reversible actives } \\
\hline \multirow{4}{*}{$150-300 \mathrm{~ms}$ following verb } & Omnibus ANOVA & & & \\
\hline & Group & 1,23 & 13.89 & $0.001^{* *}$ \\
\hline & Mat & 1,23 & 6.24 & $0.020^{*}$ \\
\hline & Group $\times$ Mat & 1,23 & 2.14 & 0.157 \\
\hline \multirow{13}{*}{$500-700 \mathrm{~ms}$ following verb } & Omnibus ANOVA & & & \\
\hline & Group & 1,23 & 16.50 & $0.000^{* *}$ \\
\hline & Mat & 1,23 & 0.42 & 0.522 \\
\hline & Group $\times$ Mat & 1,23 & 2.05 & 0.166 \\
\hline & Ant. vs. Post. ANOVA & & & \\
\hline & Group & 1,23 & 16.74 & $0.000^{* *}$ \\
\hline & Mat & 1,23 & 0.50 & 0.487 \\
\hline & Group $\times$ Mat & 1,23 & 2.22 & 0.150 \\
\hline & Group $\times$ Mat $\times \mathrm{A} / \mathrm{P}$ & 1,23 & 4.38 & $0.048^{*}$ \\
\hline & Posterior ANOVA & & & \\
\hline & Group & 1,23 & 29.18 & $0.000^{* *}$ \\
\hline & Mat & 1,23 & 0.06 & 0.803 \\
\hline & Group $\times$ Mat & 1,23 & 3.60 & 0.070 \\
\hline \multirow{4}{*}{ 250-600 ms following sentence-final noun } & Omnibus ANOVA & & & \\
\hline & Group & 1,23 & 9.52 & $0.005^{*}$ \\
\hline & Mat & 1,23 & 6.22 & $0.020^{*}$ \\
\hline & Group $\times$ Mat & 1,23 & 1.95 & 0.176 \\
\hline \multicolumn{5}{|l|}{ Sem. reversible passives } \\
\hline \multirow{13}{*}{ 250-350 ms following auxiliary verb } & Omnibus ANOVA & & & \\
\hline & Group & 1,23 & 8.68 & $0.007^{*}$ \\
\hline & Mat & 1,23 & 1.32 & 0.262 \\
\hline & Group $\times$ Mat & 1,23 & 1.08 & 0.310 \\
\hline & Ant. vs. Post. ANOVA & & & \\
\hline & Group & 1,23 & 8.73 & 0.007 \\
\hline & Mat & 1,23 & 1.21 & 0.282 \\
\hline & Group $\times$ Mat & 1,23 & 0.99 & 0.329 \\
\hline & Group $\times$ Mat $\times \mathrm{A} / \mathrm{P}$ & 1,23 & 4.28 & $0.050^{*}$ \\
\hline & Posterior ANOVA & & & \\
\hline & Group & 1,23 & 10.77 & $0.003^{*}$ \\
\hline & Mat & 1,23 & 5.40 & $0.029^{*}$ \\
\hline & Group $\times$ Mat & 1,23 & 3.22 & 0.086 \\
\hline \multirow{4}{*}{ 450-700 ms following auxiliary verb } & Omnibus ANOVA & & & \\
\hline & Group & 1,23 & 15.94 & $0.001^{* *}$ \\
\hline & Mat & 1,23 & 6.25 & $0.020^{*}$ \\
\hline & Group $\times$ Mat & 1,23 & 1.35 & 0.257 \\
\hline \multirow{4}{*}{ 400-700 ms following sentence-final noun } & Omnibus ANOVA & & & \\
\hline & Group & 1,23 & 0.37 & 0.550 \\
\hline & Mat & 1,23 & 2.65 & 0.117 \\
\hline & Group $\times$ Mat & 1,23 & 0.25 & 0.620 \\
\hline
\end{tabular}

Note. Mat: matching type (matching vs. mismatching). ${ }^{*} p \leq 0.05 ;{ }^{* *} p \leq 0.001$. Omnibus ANOVA: 29 electrodes; anterior vs. posterior ANOVA: $2 \times 10$ electrodes; posterior ANOVA: 10 electrodes. 
(A) Semantically reversible (passive)
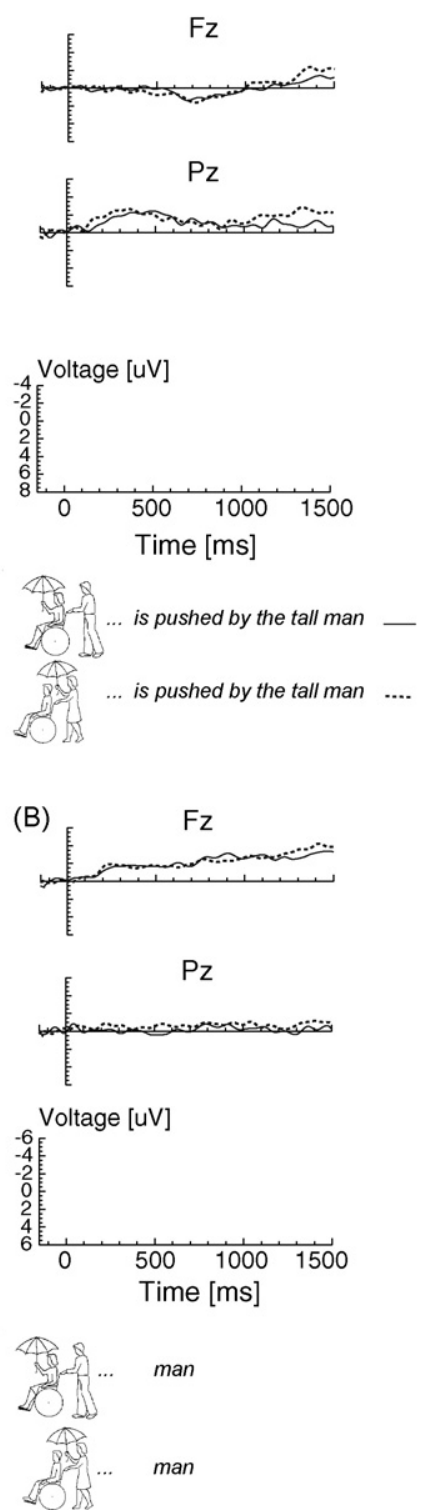

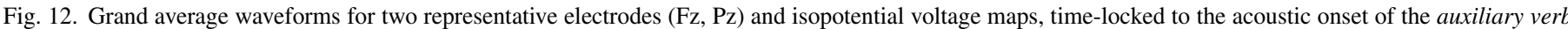

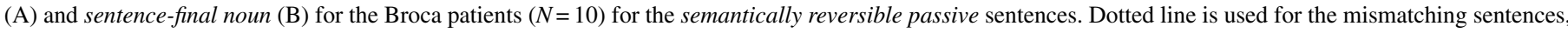

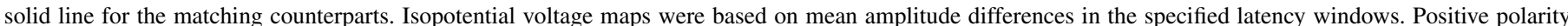

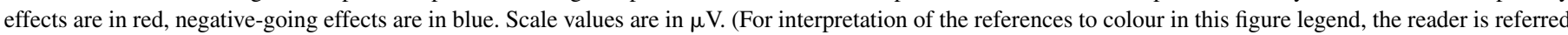
to the web version of the article.)

Table 14

Summary of ERP results

Normal controls $(N=15)$

RH patients $(N=8)$

Broca patients $(N=10)$

I: Sem. Irrev. Act.

Verb, 100-200 ms: neg. effect

Verb, 500-800 ms: pos. effect

Noun2, 250-600 ms: neg. effect

II: Sem. Rev. Act.

Verb, 150-300 ms: neg. effect Verb, 500-700 ms: -

Noun2, 250-600 ms: neg. effect

III: Sem. Rev. Pass

Aux verb, 250-350 ms: neg. effect Aux verb, 450-700 ms: pos. effect Noun2, 400-700 ms: -
Verb, 100-200 ms: -

Verb, 500-800 ms: pos. effect

Noun2, 250-600 ms: neg. effect

Verb, 150-300 ms: neg. effect

Verb, 500-700 ms: -

Noun2, 250-600 ms: neg. effect

Aux verb, 250-350 ms: -

Aux verb, 600-850 ms: pos. effect

Noun2, 400-700 ms: neg. effect
Verb, 100-200 ms: -

Verb, 800-1100 ms: pos. effect

Noun2, 250-600 ms: -

Verb, 150-300 ms:-

Verb, 500-700 ms: -

Noun2, 250-600 ms: neg. effect

Aux verb, 250-350 ms: -

Aux verb, 450-700 ms: -

Noun2, 400-700 ms: - 
input to the information in working memory. However, whereas the primary cognitive process in their experiment was a matchto-sample detection of incorrect words, our present experiment required an analysis of thematic roles.

The positive effect shift that was present for sentence types I and III in the normal control subjects (see Table 14), resembled both in terms of scalp distribution and time course a P600 effect. Recently, Kuperberg et al. (2003) found a P600 effect when subjects were presented with sentences containing thematic role animacy violations. They interpreted this P600 as a result of the discrepancy between the probable thematic role and the actual assigned thematic role. It is interesting to see that in our present study we found not only a P600 effect to the critical verb of the sentences that contained a thematic role animacy violation itself (sentence type I). A P600 effect was also found for sentences that were acceptable in itself, but contained thematic roles that were contradictory to thematic information perceived from the previously presented picture (sentence type III). The question of what process is indexed by this P600 effect cannot be directly answered from this experiment. One option is that the mismatch between the conceptual representation the subject has in mind, and the actual sentence, is the event underlying the P600 effect (Kolk et al., 2003). Another possibility is that the P600 reflects an on-line attempt to reassign thematic roles (Kuperberg et al., 2003). In this latter account, amplitude size of the P600 effect is seen as a function of how effortful the reassignment process is. This might be a tentative explanation for the fact that amplitude size of the P600 effects in the current study differed as a function of sentence type. We should, however, consider yet another explanation for the elicitation of the P600 effect. It is possible that the strong thematic bias emanating from the picture, makes it more difficult to assign grammatical roles onto sentences that are in conflict with the perceived information. In this view, the P600 effect would reflect the effortful process of assigning grammatical roles. This grammatical role assignment is harder if semantic biases are in favour of an alternative syntactic choice.

For sentence types I and II, a negative effect was elicited to the sentence-final noun. In sentence-final positions, an N400-like effect is often seen as result of a processing-problem somewhere earlier in the sentence (Hagoort, Brown, Groothusen, 1993; Osterhout \& Holcomb, 1992, 1993). Thus, local processing problems somewhere in the sentence can have a downstream effect on the amplitude of the N400-like effect to the word in sentence-final position. In our experiment there was a local processing problem at the position of the verb which resulted in local ERP effects. In addition, we found sentence-final negativities. We think that this sentence-final effect is a reflection of the following processing problem: it is more difficult to end up with a coherent verbal message in the context of contradictory visual input than in the situation where visual and verbal information agree with one another. We can offer, however, no explanation why this sentence-final negativity is absent in sentence type III.

Regardless of the precise functional interpretation of the ERP effects mentioned above, the crucial aspect of the ERP pattern in the normal controls is, that they showed sensitivity to a picture-sentence mismatch as soon as the verb was heard. This suggests that the mismatch between the representation of the presented picture and the unfolding sentence was being detected on-line. The fact that ERP effects were obtained at the position of the verb implies that, given the visual context of the picture, thematic roles were assigned immediately, thus as soon as, or in case of auxiliaries even before, the argument structure belonging to the main verb was available. Thus, subjects did not have to wait for the full lexical information that was to be associated with the thematic grid. Instead, relevant visual context (i.e. the picture) was immediately taken into account during the process of real time language comprehension. This enabled subjects to detect a picture-sentence mismatch when encountering the verb. Early integration of visual context during on-line comprehension has been demonstrated before in eye-movement studies (e.g. Spivey et al., 2002; Tanenhaus et al., 1995). Of special interest for this discussion is a recent eye-movement study of Knoeferle, Crocker, Scheepers, and Pickering (2005). They investigated whether visual role information provided by agentaction-patient events as depicted in event scenes (i.e. a picture), would guide thematic-role assignment during on-line auditory comprehension. They found anticipatory eye-movements in the event scenes to the appropriate role filler once the verb had referred to the depicted action. This was interpreted by the authors as an on-line influence of depicted events on incremental thematic role assignment. Both these eye-movement data and the ERP results of our present study support the idea that thematic role assignment is an incremental process, with early integration of visual contextual information. These features of the thematic role assignment process certainly contribute to the amazing speed with which neurologically unimpaired subjects can comprehend everyday language in the context of visual information. A cognitive architecture with a close communication between visual and language processing seems for this ability to be required.

It is interesting that for the semantically reversible passive sentences ERP effects already were obtained for the auxiliary verb, when no thematic grid is available yet. However, it is conceivable that in the context of visual information, which provides constraints on possible arguments, the information of the auxiliary verb is used to predict a (mis)match between the picture and the sentence, since one of the two arguments was already provided before the auxiliary verb. Another possibility is that the early effect is a consequence of connected speech and possible co-articulation between the auxiliary verb and the main verb. In both cases, the finding highlights the immediacy of thematic role assignment in healthy individuals.

The ERP results of the RH patients bear clear resemblance to the data of the normal controls in the sense that both groups of subjects showed sensitivity to a picture-sentence mismatch as soon as the verb was heard. Although there were some differences (see Table 14), the most important finding is that also in the RH patients thematic role assignment in the context of visual information seemed to be an immediate process.

The ERP results of the Broca's aphasics were clearly different from those of the other two subject groups (see Table 14). Whereas for both control groups ERP effects were obtained 
at the position of the verb (for all sentence conditions), the Broca's aphasics showed an ERP effect (i.e. a positive shift) time-locked to the onset of the verb only for the semantically irreversible sentences. Moreover, this effect was, when compared to the control groups, reduced and considerably delayed. For the semantically reversible active sentences, a negative ERP effect was found only for the sentence-final noun. For the semantically reversible passive sentences, no ERP effects were obtained at all. Together, these ERP data indicate that the Broca's aphasics did not show such an on-line sensitivity to the picture-sentence mismatch as displayed by the control groups, because ERP effects time-locked to the onset of the verb were either delayed (semantically irreversible active sentences) or even absent (both types of semantically reversible sentences). This suggests that the process of thematic role assignment in the context of visual information was, at the very least, not as immediate as in the healthy control subjects. The ERP pattern of the Broca patients seemed also differentially affected by semantic (ir)reversibility and syntactic complexity, with the largest deviation from the normal controls for the semantically reversible passive sentences. For this latter sentence type, no electrophysiological indication of on-line thematic role assignment was found. Before further discussing the implications of these ERP results, we will first take the off-line behavioral results of the Broca patients into consideration.

To summarize the behavioral results, Broca patients responded considerably slower than both normal controls and $\mathrm{RH}$ patients, with a tendency in the Broca patients for response latency to be longest for the reversible passive sentences. Broca patients made also far more errors than both control groups, with high error rates for the non-matching irreversible active sentences. In addition, there were a large number of errors for the matching passive sentences.

The overall longer decision times in the Broca patients cannot exclusively be ascribed to a general consequence of brain damage. Since, brain-damaged controls without aphasia, i.e. the RH patients, showed much faster RTs that could not statistically be distinguished from the normal controls. This fact, together with Broca patients' increasing RTs with increasing syntactic complexity, suggest that the longer decision times more likely are related to the underlying language processing deficit rather than an aspecific consequence of brain lesion.

The relatively high error rates for the non-matching irreversible active sentences deserve particular attention. Recall, this was the condition in which for instance a picture was presented containing a woman reading a book, while the sentence contained a selectional restriction violation "The book on this picture reads the woman". Also Saffran, Schwartz, and Linebarger (1998) reported that agrammatic comprehenders, when tested with a speeded plausibility judgement task, found it very hard to reject sentences like \#The deer shot the hunter, even though this is a simple active sentence. To explain this result, the authors proposed that semantic constraints contribute to the assignment of NPs to thematic roles in normal sentence processing, and that this influence is much stronger in Broca patients as a result of a decrease in the syntactic contribution to this process. In this respect we would like to call attention to a previous ERP study of ours (Hagoort, Wassenaar, \& Brown, 2003). In that study, Broca patients with a severe syntactic comprehension impairment showed, in response to word-order violations, an N400effect instead of a syntax-related ERP effect. Such an N400 effect is usually observed to semantic binding operations during on-line language comprehension. This result suggested that these Broca patients seemed to compensate for their syntactic deficit by relying more strongly on semantic information during on-line sentence comprehension. Returning to the present experiment, it is conceivable that, in the context of visual information, a bias in the Broca patients to accept an interpretation suggested by the semantics of the content words, although being inconsistent with the sentence's syntactic form, is even enhanced. It is interesting that also in the normal controls the highest error rates were induced by the mismatching semantically irreversible sentences. This seems to be in line with the suggestion that a strong conceptual bias can modulate or even overrule syntactic structure (cf. Kolk et al., 2003). Furthermore, Ferreira (2003) has pointed out that noncanonical sentences frequently are misinterpreted since language comprehension is often based on a shallow analysis rather than on a detailed linguistic representation of the sentences, especially when implausible ideas are expressed. A more shallow interpretation of a sentence would rather lead to an interpretation consistent with schematic knowledge. This would explain why even normal control participants found non-matching irreversible active sentences relatively hard to reject.

The relatively large numbers of errors in the Broca patients for the matching passive sentences suggest that in some cases the patients interpreted the passive sentences as actives. A similar tendency in Broca patients toward thematic reversals in the comprehension of semantically reversible passive sentences has been frequently observed. It is noteworthy that the Broca patients from our present study however still performed above chance level for the passive sentences.

To conclude, although their error rates were much higher and their response times much slower, the performance of the Broca patients was, except for the mismatching irreversible sentences, still above chance. This implies that the Broca patients showed, to a certain extent, off-line behavioral sensitivity to sentence-picture mismatches.

When we compare the on-line ERP signatures of the thematic role assignment process in the Broca patients to their off-line behavioral pattern, it is striking that there seems to be a dissociation between their on- and off-line sensitivity. That is, for both types of reversible sentences, no ERP effects at the verb's position were elicited, but the off-line performance for these sentences was still above chance. In addition, for the semantically irreversible sentences an ERP effect was found at the verb's position (although reduced and delayed) while the offline performance showed relatively high error rates. Caplan and Waters (2003) state that studies of syntactic processing by aphasic patients show often complex relationships between on- and off-line performances. They re-examined therefore the question of the relationship between on- and off-line processing of syntactic structure in aphasic patients using an auditory moving window task. The Broca patients in that study, but not the fluent 
aphasics, showed a correspondence between impaired off-line performance in syntactic comprehension and disorders in online syntactic processing. Such a correspondence suggests that in these patients on-line processing deficits underlied the observed off-line impairments.

The data of our present experiment seem to indicate that Broca patients' problems with assigning thematic roles in visual context already manifested themselves during on-line processing. Nevertheless, the off-line responses for the reversible sentences were actually better than one would have expected on the basis of their on-line ERP profile. This implies that the Broca patients were, to a certain extent, able to detect a sentence-picture mismatch. But, this detection did not happen on-line. The very long response latencies in the Broca patients make it likely that off-line response 'strategies' have been adopted to decide whether a sentence and a picture matched or not. The exact nature of these response strategies cannot be unraveled from this experiment. It is possible that, at the moment that patients were required to give their match/non-match decision, fragments of a sentence were still present as an auditory trace in working memory. Their off-line performance could be partly based on a 'strategic' consultation of the auditory trace for matching/mismatching information. For the reversible passive sentences, it is not unlikely that an 'agent-first' strategy was involved given the relatively large percentage of errors for the matching sentences.

If the syntactic contribution to the on-line language comprehension process is decreased in the Broca patients, thematic information can dominate syntactic information. Under the assumption that the P600 effect, as obtained in the control groups, reflects effortful assignment of grammatical roles in the context of contradictory thematic information, an absence of such a P600 effect in the Broca patients becomes understandable. A preponderant reliance on thematic information during on-line comprehension will prevent syntactic information to play a noticeable role. The data demonstrate furthermore that, although Broca patients are impaired in their ability to assign thematic roles in real-time, they can reduce the consequences of the deficit to a certain extent by adopting off-line response strategies. Thus, aphasic off-line performance reveals the end product of the comprehension process consisting of contributions of both residual on-line comprehension abilities and compensations (Caplan, Waters, DeDe, Michaud, \& Reddy, 2004). This implies that response patterns from classical sentence-picture matching tasks can mask the nature of underlying on-line processing deficits.

To conclude, the results of this study showed that in neurologically unimpaired individuals thematic role assignment in the context of visual information was an immediate process. This in contrast to patients with Broca's aphasia who demonstrated no signs of on-line sensitivity to the picture-sentence mismatches. The syntactic contribution to the thematic role assignment process seemed to be diminished given the reduction and even absence of P600 effects. Nevertheless, Broca patients showed some off-line behavioral sensitivity to the sentence-picture mismatches. Longer response latencies make it likely that off-line response strategies are used.

\section{Acknowledgements}

We wish to thank Clarette Blaauw and Anke Kooijman for their help in screening the neurological patients, Mariken ter Keurs for making available the CT/MRI scans, Jelle van Dijk for scanning the pictures, and Petra van Alphen for lending her voice to the spoken stimuli. This research was supported in part by grant 400-56-384 from the Netherlands Organization for Scientific Research (NWO).

\section{References}

American Electroencephalographic Society. (1994). Guidelines for standard electrode position nomenclature. Journal of Clinical Neurophysiology, 11, 111-113.

Bastiaanse, R., Maas, E., \& Rispens, J. (2000). Werkwoorden- en zinnen test. Lisse: Swets \& Zeitlinger.

Berndt, R. S., \& Caramazza, A. (1999). How "regular" is sentence comprehension in Broca's aphasia? It depends on how you select the patients. Brain and Language, 67, 242-247.

Berndt, R. S., Mitchum, C. C., \& Haendiges, A. N. (1996). Comprehension of reversible sentences in "agrammatism": A meta-analysis. Cognition, 58, 289-308.

Berndt, R. S., Mitchum, C. C., \& Wayland, S. (1997). Patterns of sentence comprehension in aphasia: A consideration of three hypotheses. Brain and Language, 60, 197-221.

Caplan, D., \& Hildebrandt, N. (1988). Disorders of syntactic comprehension. Cambridge, MA: MIT Press.

Caplan, D., \& Waters, G. (2003). On-line syntactic processing in aphasia: Studies with auditory moving window presentation. Brain and Language, 84, 222-249.

Caplan, D., Waters, G., DeDe, G., Michaud, J., \& Reddy, A. (2004). A study of syntactic processing in aphasia I: Behavioral (psycholinguistic) aspects. Brain and Language, 91, 64-65.

Caramazza, A., Capitani, E., Rey, A., \& Berndt, R. S. (2001). Agrammatic Broca's aphasia is not associated with a single pattern of comprehension performance. Brain and Language, 76, 158-184.

Caramazza, A., \& Zurif, E. B. (1976). Dissociation of algorithmic and heuristic processes in language comprehension: Evidence from aphasia. Brain and Language, 3, 572-582.

Chatterjee, A., Maher, L. M., \& Heilman, K. M. (1995). Spatial characteristics of thematic role representation. Neuropsychologia, 33, 643-648.

Chatterjee, A., Southwood, M. H., \& Basilico, D. (1999). Verbs, events and spatial representations. Neuropsychologia, 37, 395-402.

Connolly, J. F., \& D'Arcy, R. C. N. (2000). Innovations in neuropsychological assessment using event-related brain potentials. International Journal of Psychophysiology, 37, 31-47.

Connolly, J. F., D’Arcy, R. C. N., Newman, R. L., \& Kemps, R. (2000). The application of cognitive event-related brain potentials (ERPs) in languageimpaired individuals: Review and case studies. International Journal of Psychophysiology, 38, 55-70.

D'Arcy, R. C. N., \& Connolly, J. F. (1999). An event-related brain potential study of receptive speech comprehension using a modified token test. Neuropsychologia, 37, 1477-1489.

Ferreira, F. (2003). The misinterpretation of noncanonical sentences. Cognitive Psychology, 47, 164-203.

Flores D'Arcais, G. B. (1973). Some perceptual determinants of sentence construction. In G. B. Flores D'Arcais (Ed.), Studies in perception: Festschrift for Fabio Metelli (pp. 344-373). Milano: Aldo Martello-Giunti Editore.

Graetz, P., De Bleser, R., \& Willmes, K. (1992). De Akense Afasie test. Lisse: Swets \& Zeitlinger.

Gratton, G., Coles, M. G. H., \& Donchin, E. (1983). A new method for off-line removal of ocular artifact. Electroencephalography and Clinical Neurophysiology, 55, 468-484.

Grodzinsky, Y. (1986). Language deficits and the theory of syntax. Brain and Language, 27, 135-159. 
Grodzinsky, Y.(1995). Trace deletion, theta-roles, and cognitive strategies. Brain and Language, 51, 469-497.

Grodzinsky, Y. (2000). The neurology of syntax: Language use without Broca's area. Behavioral and Brain Sciences, 23, 1-71.

Grodzinsky, Y., Piñango, M. M., Zurif, E., \& Drai, D. (1999). The critical role of group studies in neuropsychology: Comprehension regularities in Broca's Aphasia. Brain and Language, 67, 134-147.

Haarmann, H. J., Just, M. A., \& Carpenter, P. A. (1997). Aphasic sentence comprehension as a resource deficit: A computational approach. Brain and Language, 59, 76-120.

Hagoort, P. (2003). The interplay between syntax and semantics during sentence comprehension: ERP effects of combining syntactic and semantic violations. Journal of Cognitive Neuroscience, 15, 883-899.

Hagoort, P., Brown, C. M., \& Groothusen, J. (1993). The syntactic positive shift (SPS) as an ERP measure of syntactic processing. Language and Cognitive Processes, 8, 439-483.

Hagoort, P., \& Kutas, M. (1995). Electrophysiological insights into language deficits. In F. Boller, \& J. Grafman (Eds.), Handbook of neuropsychology: Vol. 10,. Amsterdam: Elsevier.

Hagoort, P., Wassenaar, M., \& Brown, C. M. (2003). Real-time semantic compensation in patients with agrammatic comprehension: Electrophysiological evidence for multiple-route plasticity. PNAS, 100(7), 43404345.

Huber, W., Klingenberg, G., Poeck, K., \& Willmes, K. (1993). Die Supplemente zum Aachener Aphasie Test: Aufbau und Resultate der Validierung. Neurolinguistik, 7, 43-66.

Just, M. A., \& Carpenter, P. A. (1992). A capacity theory of comprehension: Individual differences in working memory. Psychological Review, 99, 122-149.

Knoeferle, P., Crocker, M. W., Scheepers, C., \& Pickering, M. J. (2005). The influence of the immediate visual context on incremental thematic roleassignment: Evidence from eye-movements in depicted events. Cognition, 95, 95-127.

Kolk, H. H. J., Chwilla, D. J., van Herten, M., \& Oor, P. J. W. (2003). Structure and limited capacity in verbal working memory: A study with event-related potentials. Brain and Language, 85, 1-36.

Kuperberg, G. R., Sitnikova, T., Caplan, D., \& Holcomb, P. J. (2003). Electrophysiological distinctions in processing conceptual relationships within simple sentences. Cognitive Brain Research, 17, 117-129.

Linebarger, M. (1990). Neuropsychology of sentence parsing. In A. Caramazza (Ed.), Cognitive neuropsychology and neurolinguistics: Advances in models of cognitive function and impairment. Hillsdale, NY: Erlbaum.
Linebarger, M. (1995). Agrammatism as evidence about grammar. Brain and Language, 50, 52-91.

Linebarger, M. C., Schwartz, M. F., \& Saffran, E. M. (1983). Sensitivity to grammatical structure in so-called agrammatic aphasics. Cognition, 13, 361-392.

Miyake, A., Carpenter, P. A., \& Just, M. A. (1994). A capacity approach to syntactic comprehension disorders: Making normal adults perform like aphasic patients. Cognitive Neuropsychology, 11, 671-717.

Miyake, A., Carpenter, P. A., \& Just, M. A. (1995). Reduced resources and specific impairments in normal and aphasic sentence comprehension. Cognitive Neuropsychology, 12, 651-679.

Oldfield, R. C. (1971). The assessment and analysis of handedness: The Edinburgh inventory. Neuropsychologia, 9, 97-113.

Orgass, B. (1986). Der Token test. Weinheim: Beltz.

Osterhout, L., \& Holcomb, P. J. (1992). Event-related brain potentials elicited by syntactic anomaly. Journal of Memory and Language, 31, 785-806.

Osterhout, L., \& Holcomb, P. J. (1993). Event-related potentials and syntactic anomaly: Evidence of anomaly detection during the perception of continuous speech. Language and Cognitive Processes, 8, 413-438.

Saffran, E. M., Schwartz, M. F., \& Linebarger, M. C. (1998). Semantic influences on thematic role assignment: Evidence from normals and aphasics. Brain and Language, 62, 255-297.

Schwartz, M. F., Linebarger, M., Saffran, E. M., \& Pate, D. S. (1987). Syntactic transparency and sentence interpretation in aphasia. Language and Cognitive Processes, 2, 85-113.

Schwartz, M. F., Linebarger, M. C., \& Saffran, E. M. (1985). The status of the syntactic deficit theory of agrammatism. In M. L. Kean (Ed.), Agrammatism. New York: Academic Press.

Spivey, M. J., Tanenhaus, M. K., Eberhard, K., \& Sedivy, J. C. (2002). Eye movements and spoken language comprehension: Effects of visual context on syntactic ambiguity resolution. Cognitive Psychology, 45, 447-481.

Tanenhaus, M. K., Spivey-Knowlton, M. J., Eberhard, K., \& Sedivy, J. C. (1995), Integration of visual and linguistic information in spoken language comprehension. Science, 268, 1632-1634.

Ter Keurs, M., Brown, C. M., Hagoort, P., \& Stegeman, D. F. (1999). Electrophysiological manifestations of open- and closed-class words in patients with Broca's aphasia with agrammatic comprehension: An event-related brain potential study. Brain, 122, 839-854.

Wassenaar, M. E. D., \& Hagoort, P. (1994). Off-line observatie instrument voor het onderzoeken van grammatisch taalbegrip. Unpublished manuscript. Nijmegen: Max Planck Institute for Psycholinguistics.

Zurif, E., \& Piñango, M. M. (1999). The existence of comprehension patterns in Broca's aphasia. Brain and Language, 70, 133-138. 